Monatsschr Kinderheilkd 2014 [Suppl 2]

162:204-217

DOI 10.1007/s00112-014-3205-7

๑) Springer-Verlag Berlin Heidelberg 2014

\section{Abstracts der 66. Jahrestagung der Deutschen Gesellschaft für Sozialpädiatrie und
Jugendmedizin (DGSPJ) für Sozialpädiatrie und
Jugendmedizin (DGSP)}

\section{Wissenschaftliche Leitung}

Dr. med. Carsten Wurst, Suhl

\section{Vorträge}

\section{Mundgesundheit von Menschen mit Behinderungen}

\section{DGSPJ-SY-MG-1 Mundgesundheit von Menschen mit Behinderungen von Kindes- beinen an}

Heinrich-Weltzien R. $^{1}$

'Universitätsklinikum Jena, Poliklinik für Präventive Zahnheilkunde, Jena

Karies, Kariesfolgeerkrankungen, Parodontalerkrankungen, Strukturstörungen der Zähne, dentale Traumata sowie Zahnstellungs- und Kieferanomalien im Kindesalter beeinträchtigen nachhaltig die Lebensqualität und Allgemeingesundheit von Menschen mit Behinderungen im Erwachsenenalter, wenn keine frühzeitige hochwertige zahnärztliche Betreuung erfolgt. Das therapeutische Vorgehen ist oft durch ein hohes Maß an interdisziplinärer Zusammenarbeit von zahnmedizinischen, medizinischen und nichtmedizinischen Berufsgruppen gekennzeichnet. In besonderen Fällen stellt eine Zahnsanierung in Intubationsnarkose eine ergänzende Therapieform dar, die aus medizinischen Gründen indiziert und in einem Zentrum unter stationären Bedingungen durchzuführen ist. Menschen mit Behinderungen sind daher eine Patientengruppe mit speziellen zahnärztlichen Betreuungsanforderungen. Ihre Behandlung ist zeit- und personalintensiv und wird im derzeitigen zahnärztlichen Versorgungssystem nicht adäquat vergütet. Obwohl die Mehrzahl der Patienten unter den allgemeinen Praxisbedingungen behandelt werden könnte, ist ihre zahnärztliche Versorgung aufgrund der genannten Barrieren sowie einer defizitären curricularen Ausbildung von Zahnärzten und zahnärztlichem Hilfspersonal unbefriedigend. Aufgrund des demografischen Wandels sind Gesetzgeber, Versicherungsträger wie auch (zahn-)medizinische Zentren und der einzelne Zahnarzt gefordert, sich der Versorgung dieser Klientel zu stellen, um eine gleichwertige Behandlung von Patienten mit und ohne Behinderung zu gewährleisten. Dazu bedarf es Konzepte zur Verbesserung der zahnärztlichen universitären Ausbildung und postgradualen Fortbildung in Verbindung mit gesundheitspolitischen Versorgungsaspekten.

\author{
Transition 2 - Modelle in der Sozialpädiatrie
}

DGSPJ-SY-TM-1

Modelle der Transition in der Sozialpädiatrie

Peters H. ${ }^{1}$

'RFK Mainz, KiNZ, Mainz

Alle pädiatrischen Patienten benötigen in der Adoleszenz einen geregelten Übergang vom Kinder- und Jugendarzt zum Erwachsenenmediziner (Transition). Dies gilt besonders für chronisch Erkrankte. Für sie und ihre Familien bedeutet es eine besondere Herausforderung: Lösen von einer langjährigen Bindung mit zahlreichen detaillierten Kenntnissen über den Verlauf.

Grundsätzlich werden für die Transition 4 Modelle praktiziert:

1. klassische Übergabe an weiterbetreuenden Haus- und Facharzt (Unterbrechung der Behandlungskontinuität),

2. Weiterbetreuung durch Kinder- und Jugendärzte und SPZ auch nach vollendetem 18. Lebensjahr (kein Wechsel der medizinischen und therapeutischen Betreuung, zurzeit nur auf der willkürlichen Maßgabe der Kostenträger auf der Basis von Einzelfallentscheidungen möglich),

3. Übergang über „Transitionsambulanz“ in Erwachsenambulanz/-betreuung (überlappende Betreuung während Pubertät und Adoleszenz, schrittweise Übergabe),

4. Fortsetzung der Betreuung unter einem Dach (Behandlungskontinuität mit interdisziplinärem Team, Übergabe von Pädiater an Erwachsenenmediziner, das übrige therapeutisches Team und die langjährige Vertrautheit bleiben erhalten).

Für Patienten mit geistiger und oder mehrfacher Behinderung ist Modell 4 am besten. Dies setzt allerdings voraus, dass die SSPZ und Erwachsenenambulanzen (MZEB Medizinische Zentren für Erwachsene mit Behinderung) unter einem Dach eng kooperieren.

Keywords. Transition, Sozialpädiatrie, SPZ, komplexe Mehrfachbehinderungen,

\section{Transition in der Sozialpädiatrie}

\section{DGSPJ-SY-TM-2}

Erlebte Transition - Erfahrungen von jungen Menschen im Kindernetzwerk

Schmid R. ${ }^{1}$

'Kindernetzwerk e. V., Aschaffenburg, Deutschland

Über Transition wird mittlerweile auf Kongressen, in der Politik und auch innerhalb von Verbänden viel gesprochen. Dann zumeist jedoch vorwiegend über junge Menschen, aber nicht mit ihnen zusammen. Kindernetzwerk e. V. als bundesweiter Dachverband der pädiatrischen 
Eltern-Selbsthilfe hat deshalb Jugendliche, Adoleszente und junge Erwachsene selbst gefragt, was bei Transitionsprozessen zu beachten ist, damit die Übergänge in die Erwachsenenmedizin auch gelingen.

Ausgehend von einem konkreten Fallbeispiel (Sara B.) werden in dem Vortrag generelle Punkte abgleitet, die junge Menschen für den Tranistionsprozess für elementar wichtig halten. Dazu zählen unter anderem: - „Meine Meinung zählt mehr als die meiner Eltern!“ Denn bei der Transition bin ich kein Kind mehr!

- Erwachsen werden heißt für uns auch: „Junge Menschen mit besonderem Bedarf nicht unterschätzen und nicht nur als Mensch mit Defiziten sehen! Es fehlt leider oft die Möglichkeit, sich als volles Mitglied der Gesellschaft beweisen zu können“"

Aus diesen Erfahrungen leiten sich unter anderem die folgenden Wünsche und Erwartungen ab:

- Tabuthemen wie Sexualität, Vererbung oder Schwerbehindertenausweis müssen im Transitionsprozess eine deutlich größere Rolle spielen.

- Transition sollte auch in der Regelversorgung nach vorgegebenen und in Modellen bewährten Standards als Regelleistung zur Regel werden.

Der wesentliche Wunsch lautet aber: Die erlebten Transitionserfahrungen junger Menschen wie die von Sara B. sollten in Zukunft konsequent mit einbezogen und für die gewünschten fließenden Übergänge genutzt werden.

\section{DGSPJ-SY-TM-3}

Begleitung von Kindern und Jugendlichen mit Spina bifida in das Erwachsenenalter

\section{Bredel-Geißler A.', Wollstädter C. ${ }^{2}$ \\ 'RFK Mainz, Spina bifida Ambulanz, Mainz, ${ }^{2}$ RFK Mainz, KiNZ Spina bifida Ambulanz, Mainz}

Transition in der Medizin hat eine besondere Bedeutung für Kinder und Jugendliche mit komplexen, lebenslang bestehenden Krankheiten. Die medizinische Versorgung ist kontinuierlich sicherzustellen. Die Pädiatrie als umfassend versorgende Disziplin eines Lebensabschnitts muss verlassen werden, die fachärztlich getrennten Disziplinen der Erwachsenenmedizin müssen die Behandlung weiterführen.

Ein Ablöseprozess von vertrauten Versorgungsstrukturen ist wegen der besonderen Vulnerabilität in dieser Lebensphase sensibel zu begleiten. Transition muss als Begleitung (Prozess) auf Grundlage von Standards verstanden werden. Sie ist gelungen, wenn die erwachsen gewordenen Menschen das Management ihrer Gesundheitsfürsorge bestmöglich selbst übernehmen können.

Das Gesundheitssystem muss zum Gelingen des Transitionsprozesses die institutionellen Voraussetzungen (Behandlungseinrichtungen) zur Verfügung stellen. Spina bifida als komplexe Multiorganerkrankung eignet sich exemplarisch zur Darstellung der Probleme und Erfordernisse für die Begleitung von Kindern und Jugendlichen mit Mehrfachbehinderungen ins Erwachsenenalter. Die Spina bifida-Ambulanz Mainz betreut ihre Patienten in multidisziplinärem Konzept ohne Altersbeschränkung.

Das Modell der Spina bifida-Ambulanz der Rheinhessen-Fachklinik Mainz wird in Struktur und Betreuungsangebot unter besonderer Berücksichtigung der Begleitung von Kindern und Jugendlichen unter pflegerischen und psychosozialen Aspekten vorgestellt.

Keywords. Transition, komplexe Mehrfachbehinderungen, Spina bifida, multidisziplinäres Betreuungskonzept, psychosoziale Begleitung ins Erwachsenenalter, Pflege bei Spina bifida

\section{Entwicklungsneurologie}

\section{DGSPJ-SY-EN-1}

Das Trinkverhalten im Säuglingsalter und seine prognostische Bedeutung für die neurologische Entwicklung

Enders A.

'Dr. von Haunersches Kinderspital, Entwicklungsneurologie, München

Die Beurteilung kindlicher Entwicklung sollte neben der Beurteilung von neuromotorischen und Sinnesfunktionen auch die Bereiche Regulationsverhalten, sozial-emotionale Interaktion, Kommunikation und Kognition berücksichtigen. Optimalitätskriterien unbeeinträchtigter neurologischer Entwicklung sind dabei Variabilität und Anpassungsfähigkeit.

Da die Funktion des Saugens und Schluckens die am frühesten reife Koordinationsleistung des kindlichen Nervensystems ist, kommt der Beurteilung des Trinkverhaltens im frühen Säuglingsalter auch unter neurologischen Kriterien eine zentrale Bedeutung zu. Sie erlaubt Aufschluss über taktiles Empfinden an Lippen und Zunge, selektive Aufmerksamkeit, Orientierung zum Stimulus, Promptheit im reaktiven Verhalten, Tonus und Kraft, Kraftdosierung und Anpassung an den Stimulus, Frequenz, Rhythmus und Ausdauer beim Saugen. Beim Trinken wird die körperliche Belastbarkeit ebenso beurteilbar wie die Koordination von Atmen, Saugen und Schlucken. Auch der Beziehungsaspekt zwischen Mutter und Kind ist in der Situation des Trinkens mit berücksichtigt. Saugen ist damit mehr als ein Reflex. Videobeispiele sollen dies belegen.

Das Trinkverhalten im Neugeborenenalter hat nachweislich prognostische Bedeutung für die neurologische Entwicklung der Kinder im Alter von 18 Monaten (Mizuno and Ueda, DMCN 2005; 47:299-304).

\section{DGSPJ-SY-EN-2}

\section{Das Bobath-Konzept - aktueller Stand}

\section{Munsch K!}

'HAWK Hildesheim, Fakultät Soziale Arbeit und Gesundheit, Hildesheim

Das Bobath-Konzept als etabliertes physiotherapeutisches Verfahren für Kinder mit CP hat sich seit seiner Entstehung vor mehr als 50 Jahren ständig weiterentwickelt. Die Orientierung an einem ressourcenbezogenen Verständnis kindlicher Entwicklung und den jeweils aktuellen Erkenntnissen zum motorischen Lernen und der Steuerung von Bewegung sind zentrale Bestandteile des Konzeptes. In vergleichenden Untersuchungen komplexer Therapiekonzepte für Kinder mit Cerebralparese konnten keine Unterschiede abgebildet werden, weshalb es in Studien weiterzuführen scheint, Untersuchungsgruppen anhand der Bestandteile von Interventionen zu vergleichen. Eine größere Effektivität zeigten Interventionen, die Familienzentrierung, Alltags- und Zielorientierung enthalten, die die Eigenaktivität der Kinder aufgreifen und Selbstwirksamkeit ermöglichen. Behandlungen, die passiv, direktiv oder an Bewegungsfunktionen orientiert vorgehen, zeigten langfristig ein geringeres Outcome. Diese Ergebnisse werden durch das Review von Novak et al. gestützt, die „activity-focused, goal-oriented, family-centered" als Beschreibungen von Interventionen nannten, die nach diesem Review für Kinder mit Cerebralparese zu empfehlen sind. Diese Komponenten therapeutischer Ausrichtung werden im BobathKonzept verfolgt. Aus einem Intensivtherapie-Projekt im Rahmen des Bremer Bobath-Kurses an der Prof. Hess Kinderklinik wird der aktuelle Stand des Konzeptes anhand einer Falldarstellung aufgezeigt. 


\section{DGSPJ-SY-EN-3}

\section{Das Vojta-Prinzip heute}

Harmening $M{ }^{\text {? }}$

'kbo Kinderzentrum München, Physiotherapie, München

Die von Prof. Vojta beurteilten Bewegungsmuster in der Spontanbewegung der Ontogenese sowie bei den Lagereaktionen eines Säuglings und der Dynamik der Entwicklung der neonatalen Reflexe bilden eine einzigartige Form der Frühdiagnostik von Haltungs- und Bewegungsstörungen im Säuglingsalter. Sie dient dazu frühzeitig psychomotorische Fehlentwicklungen zu erkennen, um ein Kind möglichst zeitig einer geeigneten Therapie zuführen zu können.

Die vierte Säule des Vojta-Prinzips ist die Therapie. Durch Anwendung der Reflexlokomotion können elementare Bewegungsmuster bei Patienten mit geschädigtem Zentralnervensystem und Bewegungsapparat je nach Grad der Schädigung wieder erreicht werden. Die Reflexlokomotion wird auf reflexogenem Wege aktiviert. Reflex im Sinne der Reflexlokomotion steht nicht für die Art der neuronalen Steuerung, sondern bezieht sich auf die von außen therapeutisch gesetzten Reize und deren definierte und immer gleich ,automatisch “vorhandenen Bewegungsantworten. Die Auslösung der Reflexlokomotion erfolgt überwiegend aus den drei Grundpositionen Bauch-, Rücken- und Seitenlage und aus zehn von Vojta beschrieben Auslösungszonen am Körper. Durch die Anwendung der Reflexlokomotion sollen die elementaren Bestandteile der menschlichen Aufrichtung und Fortbewegung wieder zugänglich und nutzbar werden. Diese sind:

1. das Gleichgewicht des Körpers bei Bewegungen (posturale Steuerung),

2. die Aufrichtung des Körpers gegen die Schwerkraft,

3. die zielgerichteten Greif- und Schrittbewegungen der Gliedmaßen (phasische Beweglichkeit).

Bei der Reflexlokomotion handelt es sich, um die Grundlage für jede weitere Entwicklung, indem Blockaden beseitigt oder verringert werden. Das Vojta-Prinzip kann deshalb sehr gut in ein übergeordnetes Gesamtkonzept eingebunden werden.

\section{Inklusion und Schule}

\section{DGSPJ-SY-IS-3 \\ Inklusion erlebt - Erfahrungen aus dem Stadtstaat Bremen}

\section{Zimmermann E. \\ ${ }^{1}$ Gesundheitsamt, Bremen}

„Bremische Schulen haben den Auftrag, sich zu inklusiven Schulen zu entwickeln. “Diese klare Ansage des Schulgesetzes von 2009, verbunden mit einer Neuordnung des Systems sonderpädagogischer Förderung hat den Bremer Schulen einen Entwicklungsschub auf dem Weg zu inklusiver Pädagogik verschafft, der das kleinste Bundesland eine frühere Position in der Umsetzung der "Convention of the rights of persons with disabilities" einnehmen lässt. So ist es nicht verwunderlich, dass in Bremen auch bereits besonders deutlich unerwünschte Begleiterscheinungen des Umgestaltungsprozesses ebenso wie negative Folgen von Fehleinschätzungen zu dessen Beginn sichtbar werden.

Eklatantestes Beispiel hierfür ist die 2014 erfolgte erste Revision der Normen von 2009, mit der das bereits abgeschaffte Förderzentrum für den Bereich soziale und emotionale Entwicklung wieder gegründet wird. Die mit dem Inklusionsprozess verbundene Einführung von Ganztagsschulen lässt im Wettbewerb zum Hort konzeptionelle Unterschiede zwischen Bildungs- und Jugendhilfesektor und ausstehende Ressourcentransfers von der außerschulischen zur innerschulischen Unterstützung von Kindern mit speziellen Hilfebedarfen erkennen. Die unzureichende Zahl der den Inklusionsprozess befürwortenden und dafür auch kompetenten Lehrerinnen und Lehrern fordert zur Diskussion, ob und wie weit und wie schnell Inklusion über alle Behin- derungsarten hinweg an allen Schulen eines Schulbezirks gleichzeitig verwirklicht werden kann.

Für schulärztliche Dienste entstehen unter anderem beim Übergang vom Primar- in den SEK I-Bereich Beratungserfordernisse für eine größere Zahl von Lehrern, deren Kompetenz zur Pädagogik der Vielfalt in der Vergangenheit bereits bei Linkshändern ihre Begrenzung fand.

\section{Psychische Störungen im Kleinkind- und Vorschulalter}

\section{DGSPJ-SY-PS-2 \\ Fütter- und Essstörungen im Kleinkind- und Vorschulalter}

\section{Kroll M.', Schlensog-Schuster F. ${ }^{2}$}

${ }^{1}$ Uni Leipzig, Kinder- und Jugendpsychiatrie/ Psychotherapie, Leipzig, ${ }^{2}$ Uni Leipzig, Leipzig

Die Relevanz der Diagnostik und Therapie von Fütterstörungen (FS) bei Säuglingen und Kleinkindern bzw. Entwöhnungen von Sondenernährung wurde in den letzten Jahren intensiviert untersucht. Die ICDDiagnose F98.2 „Fütterstörung im frühen Kindesalter" wird im klinischen Alltag häufig durch die US-amerikanischen Einteilung DCo-3R nach Chatoor ergänzt: FS mit Beeinträchtigung der homöostatischen Regulation, FS mit Beeinträchtigung der reziproken Interaktion, frühkindliche Anorexie, sensorische Nahrungsverweigerung, FS assoziiert mit medizinischen Erkrankungen und FS nach Eingriffen in den Gastrointestinaltrakt. Wie bei allen psychiatrischen Störungen wird zunächst die organische Beteiligung abgeklärt.

Aufgrund der günstigen Lage in einem Zentrum für Kinder- und Frauenheilkunde konnte die Universitätsklinik für Kinder- und Jugendpsychiatrie/Psychotherapie (KJPP) Leipzig seit 2008 eine Spezialisierung für Kinder mit (schweren) Fütterstörungen (FS) in enger Zusammenarbeit vor allem mit der Kinderklinik, und zusätzlich mit der Kinderchirurgie, HNO-Klinik und den Fachtherapeuten (Logopädie, Physio-, Ergotherapie, Ernährungsberatung etc.) entwickeln. Neben den Sondenentwöhnungen, z. B. bei ehemaligen Frühgeborenen und den FS infolge von Deprivation behandelt das Team individuell und häufig in mehreren Intervallen FS bei komplex retardierten Kindern mit Syndromen, zum Beispiel M. Down, oder unterschiedlichen Konstellationen von Komorbidität, zum Beispiel nach Operationen aufgrund angeborener Ösophagusatresie. In Einzelfällen besteht über entsprechend qualifiziertes Personal die Möglichkeit, Eltern parallel zu behandeln. Im Vortrag werden allgemeine Aspekte zu Fütterstörungen und die Besonderheiten unserer klinischen Erfahrungen dargestellt.

\section{DGSPJ-SY-PS-3}

\section{ADHS im Vorschulalter}

Paulus F.W. ${ }^{1}$

'Universitätsklinikum des Saarlandes, Klinik für Kinder- und Jugendpsychiatrie, Psychosomatik und Psychotherapie, Homburg/Saar

Wenngleich das Durchschnittsalter für die Diagnosestellung ADHS acht Jahre beträgt, ist ADHS doch keine typische Störung nur des Grundschulalters. Vorschulkinder mit ADHS können entlang spezifischer und alterstypischer Symptomkriterien ab vier Jahren reliabel und valide diagnostiziert werden. Im Vorschulalter bestehende ADHS verursacht bedeutsame Beeinträchtigungen, ist stabil über die Zeit und sagt spätere Verhaltensprobleme des Kindesalters voraus.

Eine frühzeitige Diagnosestellung ermögliche frühzeitige Interventionen für die Kinder und ihre Familien. Dargestellt werden Befunde zur Prävalenz (Symptomprävalenz, repräsentative Prävalenz, Prävalenz einer klinisch-psychiatrischen Versorgungsstichprobe, Prävalenz auf der Basis von Krankenkassendaten), zum Verlauf und zur Komorbidität sowie Besonderheiten der Diagnosestellung in dieser Altersgruppe. 
Für das Vorschulalter ist die Effektivität verschiedener Interventionen bei ADHS nachgewiesen. Verhaltenstherapeutisch orientierte Elterntrainings und interaktionszentrierte Eltern-Kind-Therapie (PCIT) zählen zu den evidenzbasierten nichtmedikamentösen Therapien und haben bei Vorschulkindern Vorrang gegenüber Pharmakotherapie, zu der nur wenige RCT-Studien in diesem Alter vorliegen. MPH ist bei Vorschulkindern deutlich wirksam mit mittleren bis großen Effektstärken. Dargestellt werden die Ergebnisse der „Preschool ADHD Treatment Study“ (PATS) zur MPH-Medikation und Daten zur Behandlungsprävalenz mit MPH auf der Basis von Krankenkassendaten.

ADHS im Vorschulalter ist unterdiagnostiziert und unterbehandelt. Durch frühe Interventionen könnten jedoch Leiden (des Kindes, der Eltern, der Geschwister und des Umfeldes) und Folgeprobleme (wie komorbide Störungen und Beeinträchtigungen der Teilhabe) reduziert werden.

\section{DGSPJ-SY-PS-4}

\section{Depression und Angststörungen im Vorschulalter - Befunde zu Relevanz, Symptomatik und Beeinträchtigungen}

Otto Y. ${ }^{1}$, Andreas A. ${ }^{1}$, Fuchs S. ${ }^{1}$, von Klitzing K. ${ }^{1}$, Klein A. ${ }^{1}$

${ }^{1}$ Klinik und Poliklinik für Psychiatrie, Psychotherapie und Psychosomatik des Kindes- und Jugendalters, Leipzig

Ziel der vorgestellten Studie war es, die Belastung und Beeinträchtigung von Kindern mit Depressions- und Angststörungen hinsichtlich ihrer Entwicklung und ihres familiären Umfelds im Vergleich zu Kindern ohne psychische Störungen zu untersuchen. Aus den Ergebnissen sollen Schlussfolgerungen für den Umgang mit klinischen Störungen im Vorschulalter abgeleitet werden.

Internalisierende Symptome/Störungen wurden dimensional mit dem Strengths and Difficulties Questionnaire (SDQ; Goodman, 1997; MultiInformanten-Ansatz) und kategorial mit dem Preschool Age Psychiatric Assessment (PAPA, Egger \& Angold, 2004) erhoben. Die Stichprobe bestand aus $n=93$ Kindern mit reinen Angststörungen, $n=20$ Kindern mit Depressions- und Angststörungen und $n=42$ Kindern mit subklinischen Depressionssymptomen und Angststörungen sowie $n=76$ Kindern ohne psychische Störungen.

In einem ersten Schritt wurden die Stabilität der Symptome sowie die Übereinstimmung zwischen den verschiedenen Informanten (Mütter, Väter, ErzierherInnen) überprüft. Weiterhin wurde analysiert, inwiefern Aussagen zur Gesamtbeeinträchtigung der Kinder, zu Familienadversität und Familienklima sowie zu mütterlicher Psychopathologie sich zwischen den Gruppen unterschieden.

Abschließend werden im Vortrag Implikationen für die Praxis diskutiert.

\section{Freie Vorträge}

\section{Freie Themen}

\section{DGSPJ-FV-SP-1}

Spielend gesünder? Das Online-Lernspiel „Luftikids“ als Nachschulungsmaßnahme für Kinder mit Asthma bronchiale

Puch-Kapst K.', Schmidt A. ${ }^{2}$, Greuter T. ${ }^{3}$, Möller A. ${ }^{3}$, Steiß J.O. ${ }^{2}$

'outermedia GmbH, Berlin, ${ }^{2}$ Justus-Liebig-Universität Gießen, Zentrum für Kinderheilkunde und Jugendmedizin, Pädiatrische Pneumologie und Allergologie, Gießen, ${ }^{3}$ Universitäts-Kinderkliniken, Kinderspital Zürich, Pneumologische Abteilung, Zürich, Schweiz

Einleitung. „Luftikids“ (www.luftikids.de) ist ein Online-Lernspiel zur Asthmanachschulung für Kinder. Eine prospektive Studie untersuch- te den Nutzen der vierwöchigen Nachschulung. Das Lernspiel und die Studienergebnisse werden vorgestellt.

Methodik. 53 Patienten erfüllten die Einschlusskriterien (Diagnose Asthma bronchiale, Teilnahme an einer Basisschulung, Alter 8-13 Jahre). Die Patienten erhielten für vier Wochen einen Zugang zum internetbasierten Programm „Luftkids“, Spieldauer und Spielintensität wurden selbst bestimmt. Untersucht wurde der Einfluss auf Parameter wie Symptome (Husten, Dyspnoe), Anzahl der Notfallkonsultationen, Gebrauch der Bedarfsmedikation, Anzahl der Schulfehltage, Krankheitsbewältigung, Asthmakontrolle und Lungenfunktionswerte. Die Akzeptanz des Spieles wurde anhand der Dauer der Nutzung und der Anzahl der Logins gemessen. Die Datenerhebung erfolgte in einer Eingangskontrolle vor und einer Abschlusskontrolle sechs Monate nach Beendigung der Online-Nachschulung.

Ergebnisse. Es konnten signifikante Verbesserungen bezüglich der Intensität von Asthmasymptomen wie Husten $(\mathrm{p}=\mathrm{o}, 001)$ und Dyspnoe $(p=0,007)$, eine Reduktion von Notfallkonsultationen $(p<0,005)$, des Gebrauchs der Bedarfsmedikation $(\mathrm{p}=0,002)$ und der Schulfehltage $(p=0,003)$ nachgewiesen werden. Keine Veränderungen waren bei den Lungenfunktionsparametern sichtbar. „Luftikids“ wurde innerhalb der 4-wöchigen Maßnahme durchschnittlich 302,5 Minuten genutzt. Die Anzahl der Logins betrug 14 \pm 9 . Die Kinder profitierten nach Eigeneinschätzung viel bis sehr viel, $8 \%$ gaben keinen Effekt an.

Schlussfolgerung. Das Online-Lernspiel „Luftikids“ stellt eine zeitgemäße und attraktive Form der Asthmanachschulung für Kinder dar. Auch weniger schulungsmotivierte Patienten können so erreicht werden

\section{DGSPJ-FV-SP-2}

Eine vergleichende Analyse gesundheitsbezogener Lebensqualität bei Kindern mit juveniler idiopathischer Arthritis (JIA) im und bei Kindern mit allergischem Asthma bronchiale (AAB)

Klein M.O.', Duncker N.', Ankermann T.', von Bismarck P.'

'UKSH-Campus Kiel, Allgemeine Pädiatrie, Kiel

Einleitung. Über die gesundheitsbezogene Lebensqualität (TQoL) bei Kindern mit JIA ist bislang wenig bekannt. Für die häufigste chronische Erkrankung im Kindesalter, dem $\mathrm{AAB}$, sind deutliche Einschränkungen der TQoL bekannt. Wir untersuchten die Frage, ob Kinder mit einer JIA in Dauertherapie eine vergleichbare Einschränkung derTQoL erfahren und ob die Einschätzung zwischen Patient und Elternteil gleich wahrgenommen wird.

Methode. Querschnittsstudie zur gesundheitsbezogenen Lebensqualität mittels des validierten KINDL-R-Fragebogens (sechs Items), unterschiedliche Fragebögen für 3- bis 6- bzw. 7- bis 13-jährige Kinder. Befragung der Kinder und eines Elternteils. Einschluss: Kinder mit einer JIA bzw. AAB in Dauertherapie von $>1$ Jahr. Ausschluss: Vorliegen einer akuten oder weiteren chronischen Erkrankung, schwerste kognitive Einschränkungen.

Ergebnisse. JIA 3-6: 14 Kinder (11 w, 3 m), TQoLiooTQoL MW 87,0 $\pm 6,2$.

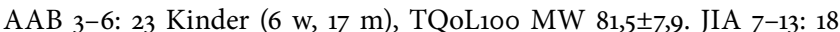
Kinder (12 w, 6 m), TQoL1oo MW 79,5 $\pm 13,8$. AAB 7-13: 34 Kinder (10 w, 24 m), TQoL10o MW 75,7 \pm SD 9,8. (Norm: 74,6). JIA Eltern 7-13: 16 Mütter, 2 Väter. TQoL10o MW 79,8 $\pm 11,2$. AAB Eltern 7-13: 33 Mütter, 1 gem., TQoLioo MW 74,6 $\pm 12,6$. (Norm: 76,9). Der TQoLioo-Vergleich liefert bei 3- bis 6-jährigen Kindern einen signifikanten Unterschied zu Gunsten der Kinder mit JIA $(t=-2,19 ; p=0,039)$. In beiden Fallgruppen 7-13 J. zeigt sich die Kinder- und Elternwahrnehmung übereinstimmend und kein signifikanter Unterschied im TQoL (Eltern: $t=-1,37 ; p=0,15$; Kinder: $\mathrm{t}=-1,06 ; \mathrm{p}=0,23)$.

Schlussfolgerung. Kinder mit JIA von 3-6 Jahren haben einen höheren TQoL gegenüber Kindern mit AAB, zwischen 7 und 13 Jahren zeigt sich eine gleichwertige Lebensqualität. In beiden Gruppen zeigt sich unter Dauertherapie ein gegenüber der Norm nicht abweichender TQoL. 
DGSPJ-FV-SP-3

Wie werden die erreicht, die es am nötigsten haben: die Rolle des öffentlichen Gesundheitsdienstes in den Münchner Mittelschulen

Franz A. ${ }^{1}$, Reincke M. ${ }^{1}$

'Landeshauptstadt München, Referat für Gesundheit und Umwelt, Gesundheitsvorsorge für Kinder und Jugendliche, München

Das Vollkonzept „Ärztin/Arzt an der Schule“ der Landeshauptstadt München ist seit dem Schuljahr 2013/14 an 6 Mittelschulen etabliert. Es beinhaltet eine wöchentliche schulärztliche Sprechstunde, Klassenuntersuchungen und Gesundheitsunterricht.

Die Auswertung der Datenerhebung vom September 2013 bis Februar 2014 aus den Sprechstunden in den Mittelschulen bestätigt, dass durch das kommunale Konzept Kinder und Jugendliche mit relevanten Versorgungslücken für eine medizinische Beratung erreicht und geeignete Maßnahmen zur (Wieder-)Eingliederung in das gesundheitliche Versorgungssystem eingeleitet werden können. 565 Vorstellungen fanden in diesem Zeitraum bei 301 Schülerinnen und Schülern statt. Der hohe Bedarf zeigt sich in der Häufigkeit der erhobenen auffälligen Befunde: körperlich $52 \%$, psychosozial $29 \%$, psychisch $19 \%$.

$\mathrm{Da}$ medizinische und psychosoziale Probleme oft gleichzeitig vorkommen und sich gegenseitig bedingen bzw. verstärken, ist eine enge Vernetzung mit anderen Berufsgruppen (z. B. Lehrern, Schulsozialpädagogen und schulpsychologischen Diensten) entscheidend für die nachhaltige Wirksamkeit der schulärztlichen Tätigkeit in der Schule. Die interdisziplinäre Zusammenarbeit ist daher ein fester Bestandteil des Konzeptes und wurde in Form von Kooperationsvereinbarungen festgeschrieben

Die Evaluation des Konzeptes trägt zur genaueren Erfassung des medizinischen und sozialen Handlungsbedarfs bei Münchner Mittelschülerinnen und -schülern bei und bietet damit die Möglichkeit zur optimierten Koordination der interdisziplinären Zusammenarbeit an der Schule.

\section{DGSPJ-FV-SP-4}

Psychische Gesundheit bei Kindern und Jugendlichen in Deutschland im zeitlichen Verlauf - Ergebnisse der BELLA LängsschnittStudie

Reiß F.', Klasen F. ', Haller A.-C.', Otto C. ${ }^{1}$, Hinzpeter B. ${ }^{1}$, Barkmann C. ${ }^{1}$, Hölling $H .{ }^{2}$, Rothenberger A. ${ }^{3}$, Herpertz-Dahlmann B. ${ }^{4}$, Döpfner M. ${ }^{5}$, RavensSieberer U.', BELLA study group

'Universitätsklinikum Hamburg-Eppendorf, Klinik für Kinder- und Jugendpsychiatrie, -psychotherapie und -psychosomatik, Hamburg, ${ }^{2}$ Robert KochInstitut, Berlin, ${ }^{3}$ Universitätsklinikum Göttingen, Institut für Kinder- und Jugendpsychiatrie und -psychotherapie, Göttingen, ${ }^{4}$ Universitätsklinikum Aachen, Klinik für Psychiatrie, Psychosomatik und Psychotherapie des Kindes- und Jugendalters, Aachen, ${ }^{5}$ Universitätsklinikum Köln, Zentrum für Neurologie und Psychiatrie, Köln

Fragestellung. Psychische Auffälligkeiten im Kindes- und Jugendalter sind häufig und stellen für Prävention und Behandlung eine gesundheitspolitische Herausforderung dar. Ziel der BELLA-Studie ist es, Entwicklungsverläufe psychischer Auffälligkeiten aufzuzeigen sowie deren Einflussfaktoren und die Inanspruchnahme von Versorgungsinstitutionen zu untersuchen.

Methoden. Die BELLA-Studie ist das Zusatzmodul zur Erfassung psychischer Gesundheit des bundesweiten Kinder- und Jugendgesundheitssurveys (KiGGS). In einer repräsentativen Unterstichprobe des KiGGS wurde eine Kohorte von Kindern und Jugendlichen zwischen 7 und 17 Jahre ( $\mathrm{N}=2863$ zur Basiserhebung) in 2003-2012 längsschnittlich vier Mal befragt. Die Erfassung psychischer Auffälligkeiten erfolgte mittels standardisierter Screeningverfahren.

Ergebnisse. Die Prävalenz psychischer Auffälligkeiten beträgt 10\% bis $11 \%$ für Kinder und Jugendliche zu allen vier Messzeitpunkten ( $\mathrm{N}=1255)$. Im zeitlichen Verlauf zeigten $74,3 \%$ der Teilnehmenden keine psychi- schen Auffälligkeiten, 7,3\% akute/wiederkehrende und 2,9\% anhaltende psychische Auffälligkeiten. Bei 15,5\% verbesserte sich der Gesundheitszustand. Die Kumulation von Risikofaktoren erhöht die Wahrscheinlichkeit der Ausbildung psychischer Auffälligkeiten, während familiäre und personale Schutzfaktoren das Risiko vermindern. In Behandlung befinden sich 63,9\% mit einer persistenten und jeder Dritte mit einer akuten/wiederkehrenden psychischen Erkrankung. Versorgungsunterschiede zeigten sich nach Wohnort (urban/ländlich sowie Ost/West) und der Stärke der Belastung.

Diskussion. Die Studie liefert aktuelle Daten zum Verlauf psychischer Gesundheit bei Kindern und Jugendlichen, zur Bedeutung familiärer Schutzfaktoren als möglichen Interventionsansatz sowie zur Versorgungsituation.

\section{DGSPJ-FV-SP-5}

Der Einfluss kognitiver und motorischer Funktionen auf die psychosoziale Adaption von Kindern und Jugendlichen mit CP und SB

\section{Kohleis K.', Storck M. ${ }^{2}$, Bode $\mathrm{H}^{3}$}

${ }^{1}$ Kinder- und Jugendambulanz/Sozialpädiatrisches Zentrum Charlottenburg-Wilmersdorf, Berlin, ${ }^{2}$ Ostfalia Hochschule für angewandte Wissenschaften, Soziale Arbeit, Suderburg, ${ }^{3}$ Universitätsklinik für Kinder- und Jugendmedizin, Sozialpädiatrisches Zentrum und Kinderneurologie, Ulm

Fragestellung. Die Ergebnisse der PACS-Studie belegen eine erhöhte Vulnerabilität von Kindern/Jugendlichen mit CP und SB für die Entwicklung psychischer Auffälligkeiten. Unsere Arbeit untersucht den spezifischen Einfluss kognitiver und motorischer Funktionen auf das psychopathologische Profil dieser Patienten.

Methode. In einer multizentrischen Querschnittstudie wurden 355 Eltern mit dem Strenghts and Difficulties Questionaire (SDQ) zu den emotionalen Problemen und Verhaltensauffälligkeiten ihrer Kinder (4-17 Jahre) befragt und mit neurologischen Daten zum Krankheitsbild komplementiert.

Ergebnisse. Erwartungsgemäß weisen Patienten mit kognitiven Beeinträchtigungen (IQ<70) signifikant höhere Prävalenzraten im SDQ-Gesamtproblemwert $(36,7 \%)$ auf als Patienten mit leicht unter- bzw. durchschnittlichen kognitiven Fähigkeiten (21,2\%). In den Subskalen des SDQ zeigen sich signifikant häufiger externalisierende Verhaltensweisen. Entgegen der Erwartung unterscheiden sich Patienten mit leichter/ mäßiger und schwerer motorischer Beeinträchtigung im SQD-Gesamtproblemwert $(28,1 \%$ vs. $25,6 \%)$ statistisch nicht bedeutsam. Deutliche Unterschiede gibt es in den Subskalen: Hyperaktivität und Prosoziales Verhalten. Diese Unterschiede sind im Wesentlichen durch die kognitiven Fähigkeiten im Sinne eines Mediator-Effektes bedingt. Insgesamt betrachtet weisen CP-Patienten mit zunehmenden Schweregrad der Erkrankung mehr externalisierende Symptome auf als Kinder mit SB.

Schlussfolgerung. Zum Verständnis der seelischen Gesundheit von Kindern und Jugendlichen mit CP und SB müssen die wechselseitigen Beziehungen neurologischer und diagnostischer Faktoren und deren Einfluss auf das psychopathologische Profil spezifischer untersucht und diskutiert werden.

\section{DGSPJ-FV-SP-6}

"Aber die wissen doch schon alles..." - Sinn und Effizienz ärztlicher Aufklärung in Schulen

Klapp C. ${ }^{1,2}$, Kramer H. ${ }^{2}$, Mais A. ${ }^{2}$, Layer C. ${ }^{2}$

'Humboldt Universität, Charité Virchow Klinik für Geburtsmedizin, Berlin, ${ }^{2}$ Ärztliche Gesellschaft zur Gesundheitsförderung ÄGGF e.V., Hamburg

Fragestellung. Jährlich kommen $>600.000$ Jugendliche in die Pubertät. Körperliche und seelische Veränderungen überfordern dann oft nicht nur sie selbst und ihr Umfeld mit zum Teil einschneidenden Folgen für Gesundheit und Lebensentwurf, wie z. B. Teenagerschwangerschaften, sexuell übertragbaren Infektionen, Depressionen, Essstörungen. STI 
sind bei jungen Menschen mit ihren oft noch nicht stabilen sexuellen Beziehungen besonders prävalent, vorwiegend Chlamydia trachomatis und HPV. Mangelnde solide Kenntnisse, gepaart mit dem jugendlichen Gefühl von Unverwundbarkeit bei Überschätzung des eigenen Wissens erhöhen das Risiko einer Infektion. Effiziente Strategien für eine wirksame Primär- und ggf. auch Sekundärprävention werden benötigt. Methode. Ärztinnen der ÄGGF e. V. (Ärztliche Gesellschaft zur Gesundheitsförderung) bieten in zwölf Bundesländern für Jugendliche in Schulen entwicklungsbegleitend strukturierte ärztliche Informationsstunden an. Aktuell werden in ca. 8000 Veranstaltungen/Jahr $>120.000$ Jugendliche erreicht. Eins der Schwerpunktthemen in der Altersgruppe der $>14$-jährigen sind STI, dabei finden neben HIV vor allem Chlamydia trachomatis und das zugehörige Screening sowie HPV-Infektionen und die Krebsprävention durch Impfung jeweils besondere Aufmerksamkeit.

Ergebnisse. Das aktive Interesse an diesem ergänzenden aufsuchenden ärztlichen Bildungsangebot ist hier bei Jugendlichen außerordentlich hoch, der Wissenszuwachs und dessen Reproduzierbarkeit signifikant. Schlussfolgerung. Die positiven Ergebnisse aus einer prospektiven, kontrollierten Interventionsstudie in verschiedenen Bundeländern, dass fokussierte ärztliche Gesundheitserziehung zum Thema Prävention bei der qua Alter und Lebenssituation „Risikogruppe für STI“ hochgradig akzeptiert ist.

\section{Trauma und Emotion}

\section{DGSPJ-SY-TE-2}

\section{Wenn Babys reden könnten ... Oder: Vier Umgangsstile für Säug-} linge von 1700 bis heute

\section{Manz F. ${ }^{1}$}

\section{${ }^{1}$ Forschungsinstitut für Kinderernährung, Dortmund}

Hintergrund. Manches, was deutsche Kinderärzte vor 100 Jahren zum Umgang mit Säuglingen empfohlen haben, erscheint uns heute nur schwer verständlich, ja beschämend wie z. B. die Operation von Babys ohne Schmerztherapie.

Material und Methoden. Literatur zum Umgang von Eltern und Ärzten mit Säuglingen aus dem 18. bis 21. Jahrhundert.

Ergebnisse. In Deutschland können in den letzten 300 Jahren 4 Umgangsstile unterschieden werden. Bis 1700 war in Deutschland ein Umgangsstil „des Nebenbei“ bei den „Wickelkindern“ üblich. Die Humanisierung der Gesellschaft durch die Aufklärung führte beim Adel und Bürgertum zu einem zärtlichen Umgangsstil bei dem die Bedürfnisse und die Würde des Babys respektiert wurden. Von 1860 bis etwa 1965 wurde ein roher Umgangsstil praktiziert. Fehlinterpretierte Befunde und der Zeitgeist führten zu einem Zerrbild vom Säugling als einem tierischen, passiven, chaotischen, triebgesteuerten, unbeseelten, wenig schmerzempfindlichen, unreifen Mängelwesen, das es vom ersten Lebenstag an zu disziplinieren galt. Die Individualität des Kindes, seine Würde und sein Interesse an selbstwirksamem Handeln wurden vernachlässigt, die zentrale Rolle der Eltern-Kind-Beziehung für die psychische Entwicklung verkannt und die Folgen körperlicher und seelischer Traumatisierungen verharmlost. Seit etwa 1965 zeigt sich ein Trend zu einem empathischen Umgangsstil, charakterisiert durch Feinfühligkeit, Kenntnisse der Säuglingspflege und anstehender Entwicklungsaufgaben, Verantwortungsbewusstsein sowie Vertrauen in die eigene intuitive elterliche Kompetenz und das Entwicklungspotential des Babys.

Schlussfolgerung. Die Geschichte lehrt wie sehr auch der Umgang mit Babys dem Zeitgeist unterworfen ist. Möge der aktuelle Trend zu einem empathischen Umgangsstil lange andauern.

\section{DGSPJ-SY-TE-3}

Professioneller Umgang mit Schoolshootings - individuelle geplante Hilfe als Entlastung für alle Betroffenen

\section{Dreiner $M{ }^{1}$}

'Zentrum für Trauma- und Konfliktmanagement, Köln

Ereignisse wie ein Schulamoklauf destabilisieren nicht nur die unmittelbar betroffenen SchülerInnen, Lehrer und Eltern, sondern vermögen auch ein Helfersystem zu mittelbar Betroffenen zu machen. Traumatische Erfahrungen sind emotional ansteckend.

Am Beispiel der Erfahrungen nach dem sog. Amoklauf in Winnenden möchte ich Ihnen aufzeigen, auf welche Weise Sie Ihre Kompetenzen effizient nutzen können, den mittelbar und unmittelbar betroffenen Eltern und ihren Kindern/Jugendlichen - bzw. dem erweiterten Familiensystem - zu helfen, ohne selbst angesteckt zu werden.

Die Erschütterungen, die solche Ereignisse erzeugen, führen oftmals dazu, dass sich eine Vielzahl von Helfern anbietet oder dass betroffene Eltern aus Sorge um das Wohl ihrer Kinder rasch professionelle Hilfe einfordern. Dies gilt sicherlich für körperliche Schäden, die möglicherweise durch einen Amoklauf entstanden sind. Die seelische Bewältigung folgt aber bestimmten Regelmäßigkeiten, die es zu beachten gilt. Die Erfahrungen haben gezeigt, dass zu frühe und zu unspezifische Unterstützung eher schadet als hilft. Hinzukommt, dass nicht jeder, der zur Zeit des Amoklaufs im Schulgebäude war auch traumatisiert ist und umgekehrt, dass z. B. Angehörige ohne anwesend zu sein eine heftige Belastungsreaktion aufweisen und dann dringend fachliche Unterstützung benötigen.

\section{Tic-Störungen und Tourette-Syndrom}

\section{DGSPJ-SY-TT-2 \\ Neue Entwicklungen in der Pharmakotherapie der Tic-Störungen}

Roessner $V^{\prime}$

'TU Dresden, Klinik für Kinder- und Jugendpsychiatrie und -psychotherapie, Dresden

Glücklicherweise wurde in den letzten Jahren mit Aripirazol ein neuer Wirkstoff für die Behandlung der Tic-Störungen auf den Markt gebracht. Die Ergebnisse der Zulassungsstudie liegen zwar noch nicht vor, aber die sehr positiven klinischen Erfahrungen haben das praktische Vorgehen bereits jetzt verändert. Zusätzlich sind durch die gestiegenen Anforderungen an Leitlinien (Stichwort $\mathrm{S}_{3}$ ) und Forschungsergebnisse zur Pathophysiologie nicht nur einige neue Antworten, sondern vor allem neue, bisher nicht diskutierte Fragen hinzugekommen. Der Vortrag gibt hier einen umfassenden und praxisorientierten Überblick zum aktuellen Stand und möglichen Entwicklungen.

\section{DGSPJ-SY-TT-3}

Alternativen zur Pharmakotherapie: von der Verhaltenstherapie bis zur Operation

\section{Müller-Vahl K.R. ${ }^{1}$}

'Medizinische Hochschule Hannover, Klinik für Psychiatrie, Sozialpsychiatrie und Psychotherapie, Hannover

Fragestellung. Seit vielen Jahren stellt die medikamentöse Behandlung von Tics mit Antipsychotika die Therapie der 1. Wahl dar. Viele Patienten sind mit dieser Behandlung allerdings unzufrieden, da Antipsychotika wie Tiaprid, Risperidon und Aripiprazol oft unzureichend wirksam sind oder zu relevanten Nebenwirkungen führen.

Material und Methode. Bereits 1973 wurde erstmals vorgeschlagen, dass auch eine Verhaltenstherapie mit Habit Reversal Training (HRT) zu einer Verminderung von Tics führt. Seither wurden zahlreiche Studien durchgeführt. Bei schwerem, Therapie resistenten Tourette-Syndrom 
(TS) wird seit einigen Jahren eine operative Therapie mittels tiefer Hirnstimulation (THS) angeboten.

Ergebnisse. An der Wirksamkeit des HRT besteht kein Zweifel, nachdem in einer großen, multizentrischen Studie ( $n=126$ Kinder) gezeigt werden konnte, dass die Behandlung im Mittel zu einer Tic-Reduktion von 30\% führt. Obwohl erstmals bereits 1999 eine THS bei einem $\mathrm{Pa}-$ tienten mit TS durchgeführt wurde, liegen bis heute lediglich Fallberichte und kleine kontrollierte Studien mit insgesamt ca. 100 Patienten vor.

Diskussion oder Schlussfolgerung. Das HRT stellt - besonders bei geringen Tics - eine sinnvolle Behandlungsalternative dar und sollte vor dem Beginn einer Pharmakotherapie stets erwogen werden. Wegen des mangelhaften Angebots steht diese Therapie allerdings nur einer Minderzahl der Patienten zur Verfügung. Eine THS sollte derzeit lediglich bei erwachsenen, schwer betroffenen und Therapie resistenten Patienten erwogen werden. Sollte die Wirksamkeit der THS in der Behandlung von Tics allerdings in den aktuell durchgeführten Studien eindeutig nachgewiesen werden, könnte auch an eine operative Behandlung von schwerstbetroffenen, ansonsten Therapie resistenten Kindern gedacht werden.

\section{Verleihung des Stefan-Engel-Preises}

\section{DGSPJ-SS-SE-1}

Deutsche Gesellschaft für Sozialpädiatrie und Jugendmedizin: 20 Jahre Zeitschrift „Kinderärztliche Praxis" und Soziale Pädiatrie

von Voss H.H.-A. ${ }^{?}$

'Kinderzentrum, München

Die „Kinderärztliche Praxis (KIPRA) ist bei ihrer Geschichte seit 1930 und über die letzten 20 Jahre zu einem Spiegelbild deutsch - deutscher Entwicklungen in der Sozialpädiatrie und Pädiatrie geworden. Als heutiges Sprachrohr der Deutschen Gesellschaft für Sozialpädiatrie und Jugendmedizin (DGSPJ) spiegelt sie wieder, auf welchen Gebieten der Kinder- und Jugendarzt in Praxis und Klinik mit aktuell bedeutsamen Themen und damit vielfachen Problemen bei Kindern u. Familien konfrontiert wird. Von Anfang an war es das Ziel der KIPRA, dass über die unverzichtbare Grundlagenforschung zur Entwicklung bei Kindern im Allgemeinen und zu Entwicklungsstörungen bei chronischen und akuten Krankheiten hinaus die Schnittstellen zwischen funktionierenden und multimodalen, wie auch interdisziplinären Konzepten in der Arbeit mit Kindern und ihren Familien mit dem Mittel qualifizierter Fortbildung weiter ausgebaut werden müssen. Die soziale Fürsorge als Auftrag für jeden Kinder- und Jugendarzt durchzieht wie ein rotes Band die Inhalte der KIPRA-Ausgaben von Anbeginn an. Ohne Zweifel hat die KIPRA Themen aufgegriffen, die den Kinderarzt zumindest in der Praxis zu 60\% seiner Arbeitszeit beschäftigen: Trennung und Scheidung, Misshandlung, Traumatisierung, Prävention, Öffentlicher Gesundheitsdienst, Impfen, Verhaltensstörungen, Sprachentwicklungsstörungen Migration, Armut, seltene Krankheiten etc.. „Neue Morbiditäten" stehen in einem engen Zusammenhang zu dem sozialen Gradienten, mit dem Kinder konfrontiert werden. Im Jahr 2003 wurde ein Vergleich bedeutsamer pädiatrischer Zeitschriften hinsichtlich ihrer Themenwahl angestellt zu ausgewählten Themen in der KIPRA (Diss.: Schabel, Anja). 1177 Publikationen wurden thematisch gewertet. Die KIPRA hat die Gesundheitsgefahren für Kinder erkannt.

\section{Sozialpädiatrie - Frühförderung - Frühe Hilfen}

\section{DGSPJ-SY-SP-3}

Die Praxispädiatrie als Zugangsweg zu den Frühen Hilfen?

Belzer F.', Kleinert L. ${ }^{1}$, Krippeit L. ', Martens Le-Bouar H. ', Barth M. ${ }^{\prime}$ 'Zentrum für Kinder- und Jugendmedizin, Universitätsklinikum Freiburg, Klinik I für Allg. Pädiatrie, Freiburg

Hintergrund. Psychosoziale Umweltfaktoren in den Lebenswelten von Säuglingen haben einen signifikanten Einfluss auf deren Entwicklung und Gesundheit. Die pädiatrischen Früherkennungsuntersuchungen eignen sich aufgrund ihrer hohen Akzeptanz in der bundesdeutschen Bevölkerung vermeintlich in besonderem Maße für eine Einschätzung von psychosozialem Hilfebedarf junger Familien anhand von gesundheitsrelevanten Umweltfaktoren.

Methoden. Der 2012 im Auftrag des Nationalen Zentrums Frühe Hilfen $(\mathrm{NZFH})$ anhand einer bundesweiten Expertenbefragung $(\mathrm{n}=41)$ entwickelte „Pädiatrische Anhaltsbogen zur Einschätzung von psychosozialem Unterstützungsbedarf ( $\mathrm{U}_{3}$ bis U6) “ wurde 2013 unter Praxisbedingungen untersucht. Fünfunddreißig niedergelassene PädiaterInnen in München und Freiburg wendeten den Anhaltsbogen über einen Zeitraum von sechs Monaten in ihrer Praxis an. Die pädiatrischen Einschätzungen hinsichtlich eines psychosozialen Hilfebedarfs wurden anschließend mit den Ergebnissen eines im häuslichen Umfeld der Familien durchgeführten Eltern-Interviews verglichen $(\mathrm{n}=161)$.

Schlussfolgerung. Es werden die Ergebnisse der Evaluation des Anhaltsbogens berichtet. Die Ergebnisse werden auf die Frage antworten, wie effektiv psychosozial belastete Familien über den Zugangsweg der Praxispädiatrie erkannt und in regional vorhandene Hilfesysteme vermittelt werden können. Darüber hinaus wird ausführlich diskutiert werden, welche von den Frühen Hilfen häufig an die Praxispädiatrie herangetragenen Erwartungen erfüllbar sind und welche nicht.

\section{Prävention in der Sozialpädiatrie}

\section{DGSPJ-SY-PI-1}

Das Bayerische Einschulungssprachscreening (BESS) - Entwicklung und Einführung eines neuen Sprachscreenings für die Schuleingangsuntersuchung in Bayern

Hölscher G. ', Stumpf P. ${ }^{2}$, Grohnfeldt M. ${ }^{2}$, Nennstiel-Ratzel U. ${ }^{1}$

'Bayerisches Landesamt für Gesundheit und Lebensmittelsicherheit, Oberschleißheim, ${ }^{2}$ Ludwig-Maximilians-Universität, Lehrstuhl für Sprachheilpädagogik und Sprachtherapie, München

Hintergrund. Sprachliche Fähigkeiten sind für einen erfolgreichen Bildungsabschluss unerlässlich. Der Erfassung des Sprachstands im Rahmen der Schuleingangsuntersuchung kommt daher eine zentrale Bedeutung zu. In Bayern wird die Schuleingangsuntersuchung im Jahr vor der Einschulung durch MitarbeiterInnen des örtlichen Gesundheitsamtes durchgeführt. Derzeit werden noch zwei Verfahren eingesetzt, die aufgrund fehlender Normierung und Validierung allgemeinen testtheoretischen Anforderungen nicht genügen. Das Bayerische Landesamt für Gesundheit und Lebensmittelsicherheit und der Lehrstuhl für Sprachheilpädagogik und Sprachtherapie der Ludwig-MaximiliansUniversität München haben daher ein neues Screeninginstrument zur Erfassung des Sprachstandes für die Schuleingangsuntersuchung in Bayern („Bayerisches Einschulungssprachscreening“, kurz BESS) entwickelt.

Methoden. Basierend auf einer ausführlichen Literaturrecherche konnten potentielle Aufgaben ausgewählt und erstellt (1o Aufgaben mit 100 Items) werden. Anschließend wurden fünf Testphasen zur Aufgabenund Itemselektion, Validierung, Test-Retestreliabilität und Normierung 
durchgeführt. Dabei wurden die Testversionen des Sprachscreenings sukzessive gekürzt und den Erfahrungen aus der Praxis angepasst. Ergebnisse. Die endgültige Version von BESS besteht aus 4 Aufgaben mit 24 Items (exkl. Lautüberprüfung). Die Durchführungszeit liegt bei ca. 7-8 Minuten. Die Normwerte sind an 3811 Kindern bestimmt und an 3810 Kindern überprüft worden.

Ausblick. Ab dem Schuljahr 2014/2015 wird das neue, standardisierte, validierte und normierte Screening flächendeckend zur Erfassung des Sprachstands von Vorschulkindern innerhalb der Schuleingangsuntersuchung in den bayerischen Gesundheitsämtern eingesetzt werden.

\section{DGSPJ-SY-PI-2}

\section{Die Identifikation des beginnenden Stotterns}

\section{Kohler J.'}

${ }^{1}$ Hochschule für Heilpädagogik, Studiengang Logopädie, Zürich, Schweiz

Hintergrund. Die Pädiatrie spielt bei der Früherkennung des beginnenden Stotterns eine wichtige Rolle. Kinderärzte müssen entscheiden, ob beobachtbare Sprechunflüssigkeiten so auffällig sind, dass eine weitere Abklärung, Beratung oder gar Therapie notwendig ist. Bisher gab es bei der Früherfassung der Redeflussstörung „Stottern“ keine empirisch abgesicherten Daten, welche kriteriengeleitet den Beratungs- bzw. Therapiebedarf erfassen würde.

Methoden. Die vorliegende Untersuchung hat ein existierendes Instrument zur Identifikation des beginnenden Stotterns hinsichtlich seiner Gütekriterien untersucht und die darin involvierten Fachpersonen (u. a. Kinderärzte und Logopädinnen) einbezogen. Es wurde in einem Retest-Verfahren die Interrater-Reliabilität bei $n=82$ Fällen bestimmt. Durch die Erfassung der tatsächlich durchgeführten Diagnostik- und Beratungstätigkeit gelang die Bestimmung von Sensitivität, Spezifität und Kriteriumsvalidität. In einem quasi-experimentellen Setting, bei dem Videos mit sprechunflüssigen Kindern den Anwendern $(\mathrm{n}=\mathbf{2 1 1}$ Fachpersonen) vorgespielt wurden, schätzten diese mit Hilfe des Instrumentes die beobachtbaren Sprechunflüssigkeiten hinsichtlich ihrer pathologischen Dimension ein. Diese Einschätzung wurde mit dem wahren Stotterschweregrad der Videofälle konfrontiert. Die so gewonnen Daten differenzierten die Gütekriterien in Abhängigkeit von der Vorerfahrung der Anwender. Stolpersteine bei der Anwendung des Instrumentes wurden durch Interviews mit den Anwendern erfasst.

Schlussfolgerung. Die Integration aller quantitativ und qualitativ erhobenen Daten lieferte die Grundlage zur Rekonstruktion des Instrumentes und zur Formulierung einer ökonomischen Best-Practice für weiterführende Entscheidungen bei Verdacht auf beginnendes Stottern.

\section{DGSPJ-SY-PI-3}

KiKuK - Kindersicherheit in Kita und Kommune. Die Herausforderung eigenverantwortlicher Lösungswege: ein konstruktivistischer Ansatz zur Förderung von Sicherheit im Kindesalter

Stumpe A. ${ }^{1}$, Böhmann J. ${ }^{2}$

'Delmenhorster Institut für Gesundheitsförderung, Delmenhorst, ${ }^{2}$ Klinikum Delmenhorst, Klinik für Kinderheilkunde und Jugendmedizin, Delmenhorst

Konstruktivistische Theorien nehmen an dass Menschen ihr Verhalten an ihren eigenen Erfahrungen ausrichten. Nur Verhaltensweisen die sich subjektiv als sinnvoll herausgestellt haben werden etabliert. Um Verhalten an die Anforderungen von Sicherheit im Kindesalter anzupassen, müssen Eltern, ErzieherInnen und kommunale Akteure das Thema zunächst wahrnehmen, ihm Bedeutung zusprechen und sich selbst als verantwortlich und wirkmächtig empfinden.

(1) „Empowerment ErzieherInnen“: In einer 3-stufigen Weiterbildung lernen ErzieherInnen Eltern für das Thema zu sensibilisieren und Verhalten zu fördern, welches zu mehr Sicherheit für ihre Kinder führt. (2) „Partizipation Eltern“: Zunächst wird die Aufmerksamkeit der Eltern mittels Fragebögen auf die häusliche Sicherheit ihrer Kinder gelenkt. Anschließend dokumentieren die Eltern vier Wochen lang alle auftre- tenden Verletzungen ihrer Kinder. Durch Feedback-Fragebögen werden reflektierte Verhaltensänderungen erfasst. (3) „Netzwerk Akteure“: Kommunale Akteure werden gebeten ihre Netzwerkpartner zu nennen und die Zusammenarbeit zum Thema Kindersicherheit zu beschreiben. Dieses Vorgehen reaktiviert das Netzwerk.

KiKuK...

- lenkt Aufmerksamkeit auf das Thema,

- leistet Hilfe zur Selbsthilfe,

- gibt Verantwortung in Kaskaden weiter,

- unterstützt und akzeptiert eigendynamische Entwicklungen.

Das Fehlen einfacher Handlungsanweisungen oder vorgefertigter Lösungen führt anfangs häufig zu Frustration. Anschließend stellt sich das Gefühl eigener Wirkmächtigkeit ein, das sich in eigenverantwortlichem Engagement äußert.

Der konstruktivistische Zugang führt zu kreativem und proaktivem Verhalten und Verantwortungsübernahme. Es scheint aussichtsreich, auch andere Projekte zur Gesundheitsförderung konstruktivistisch aufzubauen.

\section{DGSPJ-SY-PI-4}

Kinder von psychisch belasteten Eltern in Deutschland: epidemiologische Daten aus der BELLA-Studie

Haller A.-C. ${ }^{1}$, Klasen F.', Reiß F.' , Hölling H. ${ }^{2}$, Ravens-Sieberer U. ', PlaßChristl A.', BELLA study group

'Universitätsklinikum Hamburg-Eppendorf, Klinik für Kinder- und Jugendpsychiatrie, -psychotherapie und -psychosomatik, Hamburg, ${ }^{2}$ Robert Koch-Institut, Berlin

Fragestellung. Kinder von psychisch belasteten Eltern sind einem erhöhten Risiko für die Entwicklung von psychischen und verhaltensbezogenen Auffälligkeiten und Entwicklungsstörungen ausgesetzt. Dieser Zusammenhang wurde bisher nur in klinischen Stichproben belegt. Ziel dieser Studie ist es, das Risiko für psychische Störungen und Verhaltensauffälligkeiten bei Kindern psychisch belasteter Eltern anhand von Daten einer repräsentativen Bevölkerungsstichprobe zu erfassen. Methoden. Die BELLA-Studie ist das Zusatzmodul zur Erfassung psychischer Gesundheit des bundesweiten Kinder- und Jugendgesundheitssurveys (KiGGS) und untersucht seit 2003 eine für Deutschland repräsentative Stichprobe von Kindern und deren Familien. Zwischen 2009 und 2012 wurden Daten von N=3840 Kindern, Jugendlichen und jungen Erwachsenen im Alter von 3 bis 27 Jahren und deren Eltern mittels standardisierter Screeningverfahren im Hinblick auf psychische Auffälligkeiten, Risiko- und Schutzfaktoren sowie Inanspruchnahme von Versorgungsangeboten des Gesundheitssystems untersucht.

Ergebnisse. Daten von $\mathrm{N}=2436$ Familien mit Kindern im Alter von 3 bis 17 Jahren konnten für die Analysen herangezogen werden. Insgesamt wiesen $n=393$ (16,1\%) Eltern psychische Probleme auf. Das Risiko ihrer Kinder für verschiedene Störungen wird dargestellt; unter anderem waren bei diesen Kindern im Vergleich zu Kindern psychisch nicht belasteter Eltern psychische Auffälligkeiten fast vier Mal häufiger. Hinsichtlich Angststörungen hatten sie ein fast doppelt so hohes Risiko, Störungen des Sozialverhaltens waren fünf Mal häufiger.

Diskussion. Diese Ergebnisse aus einer epidemiologischen Stichprobe zeigen, dass Kinder von Eltern mit psychischen Problemen eine Hochrisikogruppe für die Entwicklung von psychischen Störungen darstellen. 
DGSPJ-WS-KF

Kinder mit Folgen nach frühen Traumata - eine Herausforderung in der kinderärztlichen Betreuung

\section{Unfried $N .^{1}$}

'Poliklinik GmbH Chemnitz - Sozialpädiatrisches Zentrum, Chemnitz

Das psychisch traumatisierte Kind in der kinderärztlichen Behandlung stellt eine Herausforderung dar. Im Workshop werden die traumabedingten, oft bizarr wirkenden Verhaltensweisen und körperlichen Symptome ohne organisch fassbare Ursache diskutiert.

Durch den Anstieg des Stresses bei bevorstehenden medizinischen Maßnahmen können alte, implizit gespeicherte Muster reaktiviert werden und die Kind-Arzt-Beziehung erheblich stören. Praktische Hilfen zur Krisenintervention sowie Maßnahmen zur Erweiterung der diagnostischen Möglichkeiten werden im mittleren Teil des Workshops erarbeitet. Anhand von Fallbeispielen können die neuen diagnostischen Wege geprüft werden.

Ziel des Workshops ist es, die durch psychische Traumatisierung entstandenen Entwicklungsblockierungen frühzeitig zu erkennen und somit lange Irrwege der Diagnostik zu vermeiden. Der Kinderarzt kann die Dissoziation bei Kindern frühzeitig bemerken und hilfreich therapeutisch intervenieren. Damit werden die in der traumatischen Erlebenszeit notwendigen Nothilfemechanismen nicht weiter fixiert und behindern eine altersentsprechende Entwicklung nicht.

\section{Poster}

\section{DGSPJ-PO-SP-1 \\ Zugang zur Kinderehabilitation: Was sich Pädiater und Eltern wünschen}

\section{Berghem S.' \\ 'Ostseestrandklinik Klaus Störtebeker, Fachklinik für Rehabilitation von Kindern und Jugendlichen, Kölpinsee}

Fragestellung. Die Zahl der durchgeführten stationären Rehabilitationsmaßnahmen für Kinder und Jugendliche sinkt seit Jahren beständig. Durch eine Befragung sollten von niedergelassenen Pädiatern und Eltern Hinweise gewonnen werden, wie der Zugang zur Rehabilitation verbessert werden könnte.

Material und Methode. Die Pädiater wurden per E-Mail eingeladen, sich an einer Befragung zur Kinderrehabilitation zu beteiligen. In einem Teil der Befragung wurden sie um Anregungen gebeten. 347 Vorschläge gingen ein. Eltern wurden über einen Aufruf im Internet gebeten, sich an einer Befragung zur Kindergesundheit und Rehabilitation zu beteiligen. 156 Eltern folgten dieser Bitte.

Ergebnisse. Pädiater wünschen sich mehr Unterstützung im Antragsprozess. Das Antragsverfahren sollte einfacher gestaltet werden, die Frage nach dem zuständigen Kostenträger sollte vereinfacht und das zweistufige Antragverfahren der GKV abgeschafft werden. Die Ablehnung von Anträgen sollte nachvollziehbar begründet werden. Auch Eltern wünschen sich mehr Klarheit, einen einfacheren Zugang zu den Antragsformularen und eine Berücksichtigung des Wunsch- und Wahlrechtes. Unterstützung erhoffen sie sich in erster Linie von ihrem Pädiater. Auch Unterstützung von anderen Fachärzten, Krankenkassen und der Rentenversicherung wird eingefordert.

Diskussion oder Schlussfolgerung. Auch wenn bereits von vielen Seiten einiges zur Prozessvereinfachung und Aufklärung unternommen wird sind die erforderlichen Informationen noch nicht in ausreichendem Maße bei den Pädiatern und Eltern von chronisch kranken Kindern angekommen. Das Antrags- und Bewilligungsverfahren sollte weiter vereinfacht und in der bereits jetzt verbesserten Form der Öffentlichkeit zugänglich gemacht werden

\section{DGSPJ-PO-SP-2}

Screening selektiver Aufmerksamkeit in der Schuleingangsuntersuchung - eine Methode zur Früherkennung von Aufmerksamkeitsstörungen?

\section{Goosmann S. ', Jochum B. ', Schölmerich A. ${ }^{2}$, Busch R. ${ }^{3,4}$, Gawehn N. ${ }^{1,5}$} ${ }^{1}$ Klinikum Dortmund gGmbH, Entwicklungsneuropsychologische Ambulanz im Sozialpädiatrisches Zentrum/Neuropädiatrie, Dortmund, ${ }^{2}$ Ruhr- Universität Bochum, Arbeitseinheit für Entwicklungspsychologie, Bochum, ${ }^{3} \mathrm{FD}$ Gesundheit des Kreis Recklinghausen, Kinder- und Jugendgesundheitsdienst, Recklinghausen, ${ }^{4}$ ehem.Gesundheitsamt der Stadt Münster, Kinder- und Jugendgesundheitsdienst, Münster, ${ }^{5}$ Hochschule für Gesundheit, Entwicklungs- und Sozialpsychologie, Bochum

Hintergrund. Zur Prävention umschriebener Entwicklungsstörungen schulischer Fertigkeiten werden in der Schuleingangsuntersuchung spezifische und unspezifische Vorläuferfähigkeiten der Kulturtechniken Lesen, Schreiben, Rechnen geprüft. Eine hervorgehobene Bedeutung hat das Screening der selektiven Aufmerksamkeit als basale Stützfunktion des Lernens (Daseking \& Petermann, 2008). Dabei ist zu beachten, dass die isolierte Durchführung neuropsychologischer Leistungstests nur in geringem Umfang zu einer reliablen/validen klinischen Diagnose beitragen kann (Drechsler, Rizzo u. Steinhausen 2009). Fragestellung. Es stellt sich die Frage, welche prädiktiven Hinweise die kindlichen selektiven Aufmerksamkeitsleistungen (SOPESS, Petermann, Daseking, Oldenhage u. Simon 2009) auf das Vorliegen von aufmerksamkeitsbezogenem Problemverhalten (FBB-ADHS-V, Döpfner, Görtz-Dorten u. Lehmkuhl 2008) geben können.

Ergebnisse. Lineare Regressionen zeigen, dass die Ergebnisse von 64 Schulanfängern im Untertest zur Erfassung der selektiven Aufmerksamkeit nur einen geringen Anteil der Varianz von aufmerksamkeitsbezogenem Problemverhalten in dem Bereich Unaufmerksamkeit aufklären können. Eine explorative Anpassung der Auswertungsmethode, welche die Leistungsgüte als Kombination aus korrekt beantworteten und fälschlich ausgelassenen Zielreizen sowie fälschlich beantworteten Distraktoren definierte, erwies sich als besserer Prädiktor für aufmerksamkeitsbezogenes Problemverhalten.

Schlussfolgerung. Es wird die Schlussfolgerung gezogen, dass das Screening selektiver Aufmerksamkeit unter Berücksichtigung weiterer Indikatoren der Leistungsgüte Hinweise auf aufmerksamkeitsbezogenes Problemverhalten geben kann. Die Notwendigkeit der Ergänzung des Leistungstests durch weitere Methoden zur klinisch bedeutsamen Vorhersage von Aufmerksamkeitsstörungen wird diskutiert.

\section{DGSPJ-PO-SP-3 \\ Säuglingsernährung aus der Sicht von Familienhebammen in Schleswig-Holstein}

Hartmann T.', Kiosz D. ', Müller M.J.'

'Institut für Humanernährung und Lebensmittelkunde, Kiel

Fragestellung. Familienhebammen haben ihren Schwerpunkt in der "psychosozialen, medizinischen Beratung und Betreuung von Familien in belastenden Lebenslagen “ im ersten Lebensjahr des Säuglings (DHV, 2011). Diese Qualifikation wird mit einer 20-tägigen Fortbildung (180 Stunden) erlangt. Pädiatrie -auch Säuglingsernährung- ist mit 16 Stunden enthalten.

Material und Methode. Um Auskunft über die Arbeitsschwerpunkte zu erhalten, wurden 43 in Schleswig-Holstein anerkannten Familienhebammen postalisch mit einem Fragebogen (21 Fragen), u. a. nach den häufigsten von Eltern gestellten Fragen und nach Ernährungsempfehlungen für den Säuglings befragt. 31 Fragebögen konnten ausgewertet werden.

Ergebnisse. Die Förderung der Mutter-Kind-Beziehung ist der wichtigste Inhalt der Betreuung (durchschnittliche Bewertung: 1,5), dicht gefolgt von der Säuglingsernährung (durchschnittliche Bewertung: 1,6). Es folgen Pflege, medizinische Beratung und Hygiene. 50\% empfehlen 
die Einführung von Beikost zwischen dem 5.-7. Lebensmonat, $43 \%$ nach dem 6.Lebensmonat, 2 Hebammen waren für individuelle Empfehlungen. Dabei ist die Lebensmittelauswahl für alle Befragten wichtiger Beratungsinhalt. Nicht empfohlen werden Honig (97\%), Nüsse ( $77 \%$ ), Hühnerei (37\%), Kuhmilch und Fisch (je 27\%).

Diskussion. Ein Grund für diese verschiedenen Vorgehensweisen ist in den Neuerungen der Empfehlungen durch Fachgesellschaften in den vergangenen Jahren zu sehen. Eine unterschiedliche Befürwortung ergibt sich auch daraus, dass die Ausbildung zur Hebamme meist viele Jahre (durchschnittlich 23 Jahre) zurückliegt.

Schlussfolgerung. Die hier dargestellten Ergebnisse machen eine stärkere Einbindung der Säuglingsernährung in die Aus- und Fortbildung von Hebammen - speziell von Familienhebammen - unter Einbezug der Pädiatrie wünschenswert.

\section{DGSPJ-PO-SP-4 \\ Familienkinderkrankenschwestern zwischen Prävention und Kinderschutz}

Bischof T.', Fischer C.', Galante-Gottschalk A.', Betz-Breuning A. ', Hamsch J.', Lutz S. ', Röhl B. ', Wieler A. ', Tropp H.-O. ', Ehehalt S.'

${ }^{1}$ Gesundheitsamt Stuttgart, Kinder- und Jugendgesundheit, Stuttgart

Hintergrund. Die Stuttgarter Familienkinderkrankenschwestern (FKKS) des Gesundheitsamtes unterstützen und begleiten Familien mit gesundheitlicher, sozialer und wirtschaftlicher Benachteiligung und spielen eine zunehmende Rolle in den Frühen Hilfen. Im Unterschied zu den Familienhebammen sind die Stuttgarter FKKS zur Fachkraft Kinderschutz weitergebildet, betreuen Kinder von der Geburt bis zur Einschulung und sind nicht an Stundenkontingente oder Überweisungsscheine gebunden.

Fragestellung. Betreuungsmerkmale der von den Stuttgarter FKKS begleiteten Familien

Methoden. Analyse der Falldokumentationen in anonymisierter Form. Ergebnisse. Die Einstufung der 70 im Jahr 2013 betreuten Familien unter Kinderschutzgesichtspunkten ergab in $26 \%$ gewichtige Anhaltspunkte für eine Kindeswohlgefährdung und in $36 \%$ mögliche Kindeswohlgefährdung; in $38 \%$ der betreuten Familien lagen keine Anhaltspunkte für eine Kindeswohlgefährdung vor. Hintergründe der Betreuung waren Unsicherheit im Umgang mit dem Kind (100\%), psychische Belastung eines Elternteils (81\%), Bindungsstörungen (60\%), mangelnde Alltagsstruktur bei der Versorgung des Kindes (54\%), Gewalterfahrung (29\%) und Suchtproblematik (23\%). Ein konkretes gesundheitliches Risiko lag bei $33 \%$ der betreuten Kinder vor. Betreut wurden überwiegend Säuglinge, fast jedes fünfte Kind war über ein Jahr alt. Gut zwei Drittel der Familien wurden länger als drei Monate begleitet, mehr als jede zehnte Familie über ein Jahr.

Schlussfolgerung. Für viele belastete Familien sind die Stuttgarter FKKS ein wichtiges Angebot im Bereich der Frühen Hilfen, besonders, wenn eine längere Betreuung benötigt wird, die Kinder schon über ein Jahr alt sind oder Kindeswohlgefährdung vorliegt.

\section{DGSPJ-PO-SP-5}

Leben ohne Lächeln. Lebensqualität und Verhaltensprobleme in Selbsteinschätzungen von Kindern und Jugendlichen mit MöbiusSyndrom

\author{
Renner G. ${ }^{1}$, Strobel L. ${ }^{2}$ \\ 'Pädagogische Hochschule Ludwigsburg, Reutlingen, ${ }^{2}$ Staatliches Seminar \\ für Didaktik und Lehrerbildung, Stuttgart
}

Fragestellung. Beim Möbius-Syndrom handelt es sich um eine seltene Störung, die durch eine kongenitale Parese der Hirnnerven VI und VII gekennzeichnet ist. Der damit verbundene fehlende mimische Gesichtsausdruck kann zu Beeinträchtigungen der sozialen Interaktion führen. Ziel der Untersuchung war die Replikation von vorliegenden
Befunden zu psychischen Beeinträchtigungen und die erstmalige Erfassung der Lebensqualität.

Methoden. 19 Kinder und Jugendliche mit Möbius-Syndrom im Alter von 9-18 Jahren bearbeiteten die Selbstberichtsformen des KINDL-R (Fragebogen zur Erfassung der gesundheitsbezogenen Lebensqualität bei Kindern und Jugendlichen) und des SDQ (Strengths and Difficulties Questionnaire). Weiter wurden syndrombedingte emotionale und soziale Belastungen erfragt.

Ergebnisse. Verglichen mit normativen Daten lagen 5,3\% bis 36,8\% der KINDL-R-Subskalenwerte und 15,8\% der Gesamtwerte im unterdurchschnittlichen Bereich (1 SD unter dem Mittelwert). Eine signifikante Diskrepanz (große Effektstärke) fand sich für die Skala Freunde. Im SDQ lagen zwischen 5,3\% und 26,3\% der Subskalenwerte und 5,3\% der Gesamtproblemwerte im grenzwertigen oder auffälligen Bereich, mit den höchsten Werten für die Skala Verhaltensprobleme mit Gleichaltrigen. Die mit dem Möbius-Syndrom verbundene Beeinträchtigung und emotionale Belastung korrelieren negativ mit dem KINDL-R-Gesamtwert.

Schlussfolgerung. Auch wenn die Selbsteinschätzungen keine generellen Hinweise auf eine reduzierte Lebensqualität oder vermehrte Verhaltensprobleme lieferten, scheint die Aufrechterhaltung befriedigender Freundschaftsbeziehungen eine besondere Herausforderung für Kinder mit Möbius-Syndrom darzustellen. Dieser Aspekt sollte daher bei der medizinischen und psychologischen Betreuung Beachtung finden.

\section{DGSPJ-PO-SP-6}

\section{Was Eltern von der stationären Rehabilitation für Kinder und} Jugendliche erwarten

Berghem S.'

'Ostseestrandklinik Klaus Störtebeker, Fachklinik für Rehabilitation von Kindern und Jugendlichen, Kölpinsee

Fragestellung. Die Zahl der durchgeführten stationären Rehabilitationsmaßnahmen für Kinder und Jugendliche sinkt seit Jahren beständig. Eine Ursache ist die rückläufige Antragsstellung. Um zu überprüfen, ob ein Grund hierfür enttäuschte Erwartungen der Eltern sind, wurden Eltern nach ihren Erwartungen befragt.

Material und Methode. Über einen Aufruf im Internet (Hinweise in Elternforen, Selbsthilfegruppen, Facebook) wurden Eltern gebeten, sich an einer Befragung zur Kindergesundheit und Rehabilitation zu beteiligen. 156 Eltern folgten dieser Bitte und füllten den Onlinefragebogen aus. Neben Fragen zum Rehazugang und zu Erfahrungen mit der Rehabilitation wurden die Eltern nach Problembereichen und Erwartungen befragt.

Ergebnisse. Als Problembereiche im Zugang zur Rehabilitation wurden neben Ablehnung durch die Kostenträger und die Hausärzte finanzielle Gründe, Heimweh der Kinder und Arbeitsplatzprobleme benannt. Eltern halten eine Lage der Rehaklinik an der See für sehr wichtig, gehen von einer fachärztlichen Betreuung, umfangreicher Diagnostik und schulmedizinischer leitliniengerechter Therapie aus. Besondere Ansprüche an die Unterkunft oder Freizeitangebote bestehen nicht, bei der Verpflegung wird Wert auf allergiegerechtes Essen und Diätformen gelegt. Etwa 10\% halten Bioverpflegung, kostenloses WLAN im Zimmer oder viele kostenlose Freizeitveranstaltungen für zwingend erforderlich oder machen die Durchführung einer Rehabilitation von solchen Faktoren abhängig.

Diskussion oder Schlussfolgerung. Die Erwartungen der meisten Eltern werden in den Fachkliniken der Kinder- und Jugendrehabilitation vollständig erfüllt. Eltern benötigen eine umfassende Unterstützung von allen Prozessbeteiligten für einen erfolgreichen Rehabilitationszugang. 


\section{DGSPJ-PO-SP-7}

Psychomotorik und Salutogenese - Salutogenetische Prinzipien in der psychomotorischen Behandlung

Greven P., ${ }^{1,2}$, Mazur I. ${ }^{1}$

'Institut für Kinder- und Jugendpsychiatrie, Psychotherapie und Soziale Pädiatrie, Berlin, ${ }^{2} \mathrm{H}: \mathrm{G}$ Hochschule für Gesundheit \& Sport, Technik \& Kunst, Fachbereich Psychologie, Berlin

Einleitung. „Psychomotorik kann als Einheit körperlich-motorischer und psychisch-geistiger Prozesse verstanden werden. (...) streng genommen gibt es gar keine Bewegung ohne Beteiligung psychischer oder geistiger Prozesse. (...) Psychomotorik ist aber auch die Bezeichnung für ein pädagogisch-therapeutisches Konzept, das die Wechselwirkung psychischer und motorischer Prozesse nutzt." (Zimmer 2006).

Fragestellung. Im Lauf der letzten Jahrzehnte hat die Psychomotorik als ursprünglich pädagogisches Förderkonzept einen deutlichen Wandel durchlaufen hin zu einem inzwischen auch gerade in der Behandlung von Kindern häufig eingesetzten therapeutischen Konzept. Meist erfolgt psychomotorische Behandlung dabei in einem multimodalen Kontext in multiprofessionellen Teams. Die Evaluation dieses therapeutischen Konzeptes steht allerdings weit hinter der Häufigkeit ihres Einsatzes zurück.

Ergebnisse. Ausgehend von Antonovsky's Konzept der „Salutogenese“ zeigt dieser Beitrag auf, wie salutogenetische Prinzipien in der psychomotorischen Behandlung wirksam werden. Dabei spielt insbesondere das sogenannte „Kohärenzgefühl“ mit seinen drei Komponenten „Sense of Comprehensibility" (Verstehbarkeit), "Sense of Manageability“ (Machbarkeit) und "Sense of Meaningfulness" (Sinn/Bedeutung) eine ganz wesentliche Rolle. Die Umsetzung dieser Prinzipien in der psychomotorischen Behandlung von Kindern und Jugendlichen wird an konkreten Beispielen dargelegt.

Schlussfolgerung. Nach der klinischen Erfahrung können insbesondere psychomotorische Gruppenangebote in einem multimodalen Behandlungssetting eine sinnvolle Ergänzung der Behandlung sowohl körperlicher als auch sozial-emotionaler Entwicklungsbeeinträchtigungen und -störungen darstellen.

\section{DGSPJ-PO-SP-8}

\section{Ressourcen bei Kindern und Jugendlichen - ein Gruppenvergleich}

Schlottbohm E.', Lindner M.', Tagay S.'

${ }^{1}$ Kliniken/Institut der Universität Duisburg-Essen, Essen

Hintergrund. Schon früh können Ressourcen vor psychischer Belastung schützen und die Widerstandsfähigkeit gegen Belastungen erhöhen, sodass sich junge Menschen, trotz schwieriger Umstände in Kindheit und Jugend, zu leistungsfähigen Erwachsenen entwickeln können. Ziel dieser Studie ist es die Ressourcenausstattung von somatisch erkrankten, psychisch erkrankten und gesunden Schülern zwischen 12 und 17 Jahren zu überprüfen und zu vergleichen.

Methode. Die Ressourcen wurden mithilfe des Essener Ressourcen Inventars für Kinder und Jugendliche (ERI-KJ) überprüft. Es wurden 104 Schüler, 49 Psychotherapiepatienten und 74 Diabetes-Patienten befragt. Das Durchschnittsalter der Gesamtstichprobe liegt bei 14,71 Jahren.

Ergebnisse. Beim Gruppenvergleich konnte ein signifikanter Unterschied bezüglich der Ressourcenausprägung zwischen den Gruppen festgestellt werden $(\mathrm{F}=33.261 ; \mathrm{p}<0,001)$. Die Psychotherapiepatienten $(M=74,89 ; S D=16,96)$ erzielten einen deutlich niedrigeren Gesamtwert im ERI-KJ als die Schüler $(\mathrm{M}=92,61 ; \mathrm{SD}=12,34)$ und die Diabetespatienten $(M=93,24 ; S D=10,98)$. Letztere zeigten den höchsten Wert im ERI$\mathrm{KJ}$, der sich jedoch nicht signifikant von dem der Schüler unterschied $(\mathrm{F}=0,11 ; \mathrm{p}=0,736)$.

Schlussfolgerung. Unsere Ergebnisse demonstrieren eindrücklich, dass es vor allem im Sinne der Prävention gilt, die Ressourcen von psychisch belasteten Kindern und Jugendlichen zu stärken, um langfristig ihre Resilienz zu fördern und psychischen Problemen vorzubeugen. Wäh- rend einer Behandlung sollte ein wesentlicher Fokus auf die Ressourcenaktivierung gelegt werden. Entgegen den Erwartungen zeigten die Diabetespatienten den höchsten Wert im ERI-KJ. Zukünftige Forschungsvorhaben sollten sich daher auch dem Zusammenhang zwischen Diabetes und Ressourcen bei Kindern und Jugendlichen widmen.

\section{DGSPJ-PO-SP-9}

\section{Schulärztliche Aufgaben zum Ende der Schullaufbahn, Einblick in} die Werkschulen Bremens

\section{Oltmann M.', Wichmann U.', Sardowski G.' \\ 'Gesundheitsamt Bremen, KJGD, Bremen}

Fragestellung. Für einige leistungsschwache Schülerinnen und Schüler ist die erfolgreiche Beendigung der Schullaufbahn in der Regelschule nicht möglich. Die seit 2009 in Bremen etablierten Werkschulen bieten diesen SchülerInnen durch das duale Konzept von Theorie und Praxis die Chance einen (erweiterten) Hauptschulabschluss zu erreichen. Wie trägt der Kinder- und Jugendgesundheitsdienst zum Erfolg der SchülerInnen bei?

Methoden. Der Kinder- und Jugendgesundheitsdienst Bremen untersucht in jedem Schuljahr die Eingangsklassen im Hinblick auf medizinische Risiken, die im gewählten Berufsfeld (z. B. Technik, Dienstleistung, Wirtschaft) zu bedenken sind. Neben einer Arbeitsschutzuntersuchung werden das individuelle somatische und psychosomatische Gesundheitsrisiko, Rauch-, Trinkgewohnheiten und Drogenkonsum erfasst. Außerdem wird die körperliche Aktivität in der Freizeit erfragt. Seit dem Jahrgang 2013/14 findet auch eine zahnärztliche Befundung im Rahmen der Werkschuluntersuchung statt.

Ergebnisse. Im Vergleich zu ihren Altersgenossen haben WerkschülerInnen häufiger gesundheitliche Probleme und psychosomatische Beschwerden, leiden verstärkt unter Übergewicht und zeigen häufiger ein gestörtes Körperbild mit einem höheren Risiko eine Essstörung zu entwickeln. Außerdem neigen sie öfter als ihre Altersgenossen zu gesundheitsschädlichem Verhalten.

Schlussfolgerung. Die Werkschuluntersuchung bietet die Möglichkeit, Schülerinnen und Schüler auf einem alternativen Weg zum Schulabschluss, individuell und auf ihr Gesundheits- und Risikoprofil abgestimmt zu beraten, um Abbrüche oder Misserfolge auf diesem Bildungsweg zu vermeiden. Unterstützend sollten zusätzlich noch Prävention und Gesundheitsförderung in den Lehrplan der Werkschulen bindend verankert werden.

\section{DGSPJ-PO-SP-10}

Die KgAS-Studie: kurz- und langfristige Veränderungen des Verhaltens und der Lebensqualität nach ambulanter Adipositasschulung für Kinder und Jugendliche und ihre Eltern

\section{Torbahn G. ${ }^{1}$, Gellhaus I. ${ }^{2}$, Koch B. ${ }^{3}$, Eberding A. ${ }^{4}$, Fink K. ${ }^{5}$, Holl R. ${ }^{5}$, van Egmond-Fröhlich A. ${ }^{6}$}

'Universität Wien, Institut für Ernährungswissenschaften, Wien, Österreich, ${ }^{2}$ St. Vincenz-Krankenhaus, Klinik für Kinder- und Jugendmedizin, Paderborn, ${ }^{3}$ Zentrum für Adipositasschulung Bremen-Stadt, Bremen, ${ }^{4}$ Christliches Kinderhospital Osnabrück, AdiFit, Osnabrück, ${ }^{5}$ Universität Ulm, Institut für Epidemiologie und medizinische Biometrie, Ulm, ${ }^{6}$ Preyer'sches Kinderspital SMZ-Süd, Wien, Österreich

Hintergrund. Die Konsensusgruppe Adipositasschulung für Kinder und Jugendliche e. V. verfasste ein Programm-Handbuch für die ambulante, interdisziplinäre Gruppenschulung „leichter, aktiver, gesünder“, das im Rahmen der KgAS-Studie evaluiert wurde. Anthropometrische und medizinische Ergebnisse werden separat dargestellt.

Methode. 303 Patienten und ihre Eltern nahmen an 14 Zentren am einjährigen Programm während der Studie teil. Veränderungen wurden durch umfassende Fragebögen (Kinder und Erwachsene) zu Therapie- 
beginn (To) und -ende ( $\mathrm{T}_{1}$ ), sowie 1 Jahr Follow-up ( $\left.\mathrm{T}_{2}\right)$ ermittelt und durch einen Fitnesstest ergänzt.

Ergebnisse. Der Konsum problematischer Nahrungsmittel (NM) sinkt*, der günstiger NM steigt $t^{*}$ kurzfristig. Essverhalten ${ }^{* * *}$, kognitive Esskontrolle ${ }^{\star * \star}$ und Familienessregeln ${ }^{\star * \star}$ verbessern sich. Häuslicher Vorrat problematischer NM, Sättigungsvolumen, Craving und Binges nehmen $\mathrm{ab}^{* * *}$. Bis auf den Verzehr gesunder NM bleiben die Veränderungen zu T2 erhalten. Die Fitness (6-Minuten-Lauf ${ }^{* * *}$, Ein-Bein-Stand ${ }^{*}$, Sit-ups ${ }^{*}$, Seitsprung ${ }^{* * *}$ ) verbessert sich bei kurz ${ }^{\star}$ - und langfristig ${ }^{\star * *}$ gesteigerter Aktivität. Die negative Ergebniserwartung bzgl. Aktivität und Sport verringert sich, ebenso wie der Fernsehkonsum ${ }^{\star}$ zu T1. Verbesserungen der Lebensqualität ${ }^{* *}$ und der psychischen Gesundheit ${ }^{* * *}$, sowie des unterstützenden Erziehungsverhaltens (bzgl. Monitoring ${ }^{* *}$ und eigenem Vorbild ${ }^{\star}$ ), lassen sich kurz- und langfristig nachweisen.

Schlussfolgerung. Für sämtliche Behandlungsziele konnten Verbesserungen belegt werden. Die Ergebnisse bestätigen das KgAS-Programm, weisen aber auch auf Bereiche zur weiteren Optimierung hin. Es bleibt zu prüfen, ob eine gezielte, familienorientierte Nachsorge die langfristigen Erfolge weiter steigern kann.

${ }^{*} \mathrm{p}<0,1 ;{ }^{* *} \mathrm{p}<0,05 ;{ }^{* *} \mathrm{p}<0,001$

\section{DGSPJ-PO-SP-11}

Eine vergleichende Analyse gesundheitsbezogener Lebensqualität bei Kindern mit juveniler idiopathischer Arthritis (JIA) im und bei Kindern mit allergischem Asthma bronchiale (AAB)

\section{Klein M.O.', Duncker N.', Ankermann T.', von Bismarck P. ${ }^{1}$ 'UKSH-Campus Kiel, Allgemeine Pädiatrie, Kiel}

Einleitung. Über die gesundheitsbezogene Lebensqualität (TQoL) bei Kindern mit JIA ist bislang wenig bekannt. Für die häufigste chronische Erkrankung im Kindesalter, dem AAB, sind deutliche Einschränkungen der TQoL bekannt. Wir untersuchten die Frage, ob Kinder mit einer JIA in Dauertherapie eine vergleichbare Einschränkung der TQoL erfahren und ob die Einschätzung zwischen Patient und Elternteil gleich wahrgenommen wird.

Methode. Querschnittsstudie zur gesundheitsbezogenen Lebensqualität mittels des validierten KINDL-R-Fragebogens (sechs Items), unterschiedliche Fragebögen für 3- bis 6- bzw. 7- bis 13-jährige Kinder. Befragung der Kinder und eines Elternteils. Einschluss: Kinder mit einer JIA bzw. AAB in Dauertherapie von >1 Jahr. Ausschluss: Vorliegen einer akuten oder weiteren chronischen Erkrankung, schwerste kognitive Einschränkungen.

Ergebnisse. JIA 3-6: 14 Kinder (11 w, 3 m), TQoL1oo TQoL MW 87,0 $\pm 6,2$. AAB 3-6: 23 Kinder (6 w, 17 m), TQoL10o MW 81,5 $\pm 7,9$. JIA 7-13: 18 Kinder (12 w, $6 \mathrm{~m}$ ), TQoL10o MW 79,5 $\pm 13,8$. AAB 7-13: 34 Kinder (10 w, 24 m), TQoL1oo MW 75,7 \pm SD 9,8. (Norm: 74,6). JIA Eltern 7-13: 16 Müt-

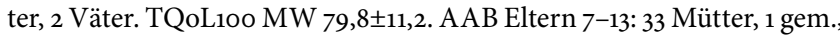
TQoL1oo MW 74,6 $\pm 12,6$. (Norm: 76,9). Der TQoLioo-Vergleich liefert bei 3- bis 6-jährigen Kindern einen signifikanten Unterschied zu Gunsten der Kinder mit JIA $(t=-2,19 ; p=0,039)$. In beiden Fallgruppen 7-13 J. zeigt sich die Kinder- und Elternwahrnehmung übereinstimmend und kein signifikanter Unterschied im TQoL (Eltern: $\mathrm{t}=-1,37 ; \mathrm{p}=0,15$; Kinder: $t=-1,06 ; p=0,23$ ).

Schlussfolgerung. Kinder mit JIA von 3-6 Jahren haben einen höheren TQoL gegenüber Kindern mit AAB, zwischen 7 und 13 Jahren zeigt sich eine gleichwertige Lebensqualität. In beiden Gruppen zeigt sich unter Dauertherapie ein gegenüber der Norm nicht abweichender TQoL.

\section{DGSPJ-PO-SP-12}

\section{Transitionssprechstunde als Bereicherung für Neuropädiater und} Neurologen - ein Fallbeispiel

\section{Thiels C. , Kleiter I. ', Salmen A. ${ }^{2}$, Köhler C. , Weigt-Usinger K. ', Lücke T.}

${ }^{1}$ Universitätsklinik für Kinder- und Jugendmedizin der Ruhr-Universität Bochum, Neuropädiatrie und Sozialpädiatrie, Bochum, ${ }^{2}$ Universitätsklinik für Neurologie der Ruhr-Universität Bochum im St. Josef-Hospital, Neurologie, Bochum

Hintergrund. Ungeklärte (neuroimmunologische) Fälle sind unbefriedigend für Patient und den betreuenden Arzt. Oftmals ist dann eine Diagnose erst nach einem über das 18. LJ hinausreichenden Beobachtungszeitraum zu stellen. In solchen Fällen profitieren Patient und betreuende Ärzte vor und nach dem 18. LJ von einer Transitionssprechstunde (TSP).

Fallbericht. 10-jähriger Junge. Cephalgien und Leistungsabbau über 4 Wochen. Innerhalb weniger Tage Meningismus, Wesensveränderung, Ataxie, Hemiparese rechts und Blasenentleerungsstörung. Diagnose: ADEM. Liquor: Protein 53 mg/dl, OKB (+), cMRT: T2- und FLAIR-Signalanhebung des gesamten subkortikalen und geringer periventrikulären Marklager, Medulla oblongata, Pons, Kleinhirnschenkeln sowie im Mittelhirn. Spinales MRT: T2-HWS-Bereich langstreckige Signalanhebung, diffus langstreckig $\mathrm{TH}_{5}-\mathrm{TH}$. Unter Methylprednisolon (MP) komplette Restitutio ad integrum. Radiologische Kontrollen über 2 Jahre Regredienz der Befunde. Sieben Jahre später bds. Optikusneuritis. Liquor: o. B., OKB und MRZ-Reaktion (-); cMRT: T2- und FLAIR-Sequenz $3 \mathrm{~mm}$ Signalanhebung rechts frontobasales Marklager, Chiasmanaher Abschnitt rechts gering; spinales MRT: Halsmark: über 3,5-4 WK ausgedehnte Signalanhebung in T2 und Höhe BWK 6/7 sowie BWK 7 - BWK 9; AQP4-AK und Anti-MOG-AK (-). VEP und OCT nicht möglich. Therapie mit MP 2-mal über 3 Tage $1 \mathrm{~g} / \mathrm{tgl}$. Visusbesserung auf jeweils 0,67 . Diskutiert wurden Neuromyelitis optica und juvenile MS. Unter GLAT bis heute, 20-jährig, keine weiteren Probleme. Nach dem 18. LJ Anti-MOG-AK (+), AQP4-AK (-). Eine NMO ist sehr wahrscheinlich.

Schlussfolgerung. Von einer TSP profitieren Patienten und das ärztliche Team. Der übergreifende Austausch fundiert und erweitert diagnostische und therapeutische Überlegungen.

\section{DGSPJ-PO-SP-13}

Gewaltprävention durch niedrigschwellige entwicklungspsychologische Beratungen belasteteter Mütter mit Kindern von 0 bis 3 Jahren

Brandi D.'

'Von Anfang an.e. V., Beratungspraxis, Hamburg

Fragestellung. Wie finden stressbelastete Mütter mit eigenen Gewalterfahrungen frühzeitig den Weg in eine entwicklungspsychologische Beratung, um ihre reflektiven Fähigkeiten zu entwickeln und ihrem Baby zur Verfügung zu stellen?

Methoden. Der Verein „Von Anfang an.e.V.“ finanziert präventive entwicklungspsychologische Eltern-Kindberatungen in Hamburg. Ergebnisse der Evaluation aus dem Jahr 2010 durch das psychologische Institut des UKE, in der Mittelschichtmütter von Babys mit Anpassungs- und Regulationsstörungen in der präventiv arbeitenden Beratungspraxis mit Fragebögen zur Beziehungsfähigkeit, zur körperlichen und seelischen Belastung der Mütter und Auswirkungen auf die intuitiven und reflektiven Fähigkeiten untersucht wurden, werden seit 2012 ausgeweitet auf Einzelberatungen bei traumatisierten und belasteten Müttern in Eltern-Kind-Zentren im Großraum von Hamburg. Im Rahmen des dort von allen Müttern gern genutzten Elternfrühstücks ergeben sich durch die Sozialpädagogen vermittelt niedrigschwellig entwicklungspsychologische Einzelberatungen. Der medizinische Hintergrund der Therapeuten des Vereins „Von Anfang an.e.V.“ erleichtert 
den frühen Vertrauensaufbau zu den Müttern, die durch Fragen zur Pflege und zum Umgang mit ihren Kindern offen sind.

Ergebnisse. Fallbeispiele psychosozial belasteter Mütter mit Kleinkindern, die durch „ruppiges" Verhalten zunächst auffällig wurden, illustrieren die präventive Wirkung niedrigschwelliger Zugänge zu entwicklungspsychologischen Beratungen bei aggressiven Entwicklungen.

Diskussion. Die Wirksamkeit von niedrigschwelligen präventiven entwicklungspsychologischen Beratungen in belasteten Familien hinsichtlich ihrer Auswirkungen auf die Symptome der Kinder und als Möglichkeit zur Gewaltprävention bei Kleinkindern wird diskutiert.

\section{DGSPJ-PO-SP-14}

Coaching von Pflegeberatern im Kinderfallmanagement - innovativer sozialmedizinischer Lösungsansatz zur besseren Versorgung von pflegebedürftigen Kindern mit komplexen Erkrankungen

\section{Schefels J.', Tourountza-Schefels E. ${ }^{2}$, Graw U. ${ }^{3}$}

${ }^{1}$ MDK Nordrhein, Duisburg, ${ }^{2}$ Akademie für Gesundheitsberufe, Wuppertal, ${ }^{3}$ Universitätsklinikum Essen-Duisburg, Essen

Hintergrund. Krankenversicherungen sind laut $₫ 12$ im SGB V verpflichtet, die medizinische Versorgung wirtschaftlich, ausreichend und zweckmäßig zu gestalten. Gesetzliche Vorgaben stoßen bei komplex kranken, technologieabhängigen Kindern an Grenzen. Es handelt eben nicht um bekannte Volkskrankheiten, sondern um ungeliebte, emotional belastende Einzelfälle, die viel Fingerspitzengefühl erfordern.

Methoden. Um diese Familien besser zu unterstützen, entwickelte der Medizinische Dienst der Krankenversicherung Nordrhein mit der NOVITAS BKK ab 2009 ein „Kinderfallmanagement“ für komplex kranke Kinder (KFM) nach dem bio-psycho-sozialen Gesundheitsmodell ICF. Die strukturierte Begleitung mit Pflegeberaterinnen (PB) bildete das Kernstück. Acht PB nahmen mit Elterneinverständnis an pädiatrischen Hausbesuchen (gesetzliche Pflegeversicherung) teil. Die PB wurden umfassend theoretisch geschult. In 2 Konferenzen pro Monat besprachen die PB und Mitarbeiter verschiedener Abteilungen der Kasse unter pädiatrischer Moderation 5 Hochkosten-Fälle und erstellten abteilungsübergreifend am „runden Tisch“ Zielvorgaben und Verlaufsdokumentationen. Es wurden Bearbeitungspfade entwickelt, um kritische Fallkonstellationen (unzureichende/inplausibele Versorgung, kasseninterne Probleme, Widersprüche) zu identifizieren. Eltern wurden regelmäßig um Rückmeldung gebeten. Die PB nutzen ab o1/2013 die Möglichkeit einer psychologischen Supervision. Die Bearbeitungszeit stieg von 2,8 auf 4,3 h pro Fall. Die Gewährung von Leistungen lief mit den PB als „Lotsen“ und primäre Ansprechpartner schneller und zielgenauer.

Schlussfolgerung. Von dem Projekt profitierten bislang 250 komplex kranke Kinder und auch die Kasse, da Doppelversorgungen, Überschneidungen, bürokratischer Aufwand für Mehrfachanfragen wegfielen und die Widerspruchsrate erheblich sank.

\section{DGSPJ-PO-SP-15}

Ergebnisse der 2-Jahres-Untersuchung ehemaliger Frühgeborener mit einem Geburtsgewicht $<1500 \mathrm{~g}$ mittels BSID II an einem kleinen Perinatalzentrum Level 1

Wurst C. ${ }^{1}$, Wiedemann F.', Jäger A. ${ }^{\text {' }}$

'SRH Zentralklinikum Suhl GmbH, Sozialpädiatrisches Zentrum, Suhl

Fragestellung. Korrelieren die Ergebnisse der 2-Jahres-Nachuntersuchung ehemaliger Frühgeborener mit einem Geburtsgewicht $<1500 \mathrm{~g}$ an einem kleinen Perinatalzentrum mit denen großer Zentren? Methodik. 103 Frühgeborene mit einem Geburtsgewicht $<1500$ g wurden mit korrigiertem 2. Lebensalter (24 \pm 1 Monate) zur Nachuntersuchung am SPZ am SRH Zentralklinikum Suhl schriftlich einbestellt. Die standardisierte Untersuchung erfolgte mittels den BSID II. Der Untersuchungszeitraum lag zwischen dem 01.03.2008 und 31.03.2014.
Ergebnisse. 67 von 103 Kindern (65,1\%) erschienen zur BSID-II-Nachuntersuchung, davon waren 42 Mädchen (62,7\%) und 25 Jungen (37,3\%). Das Gestationsalter der untersuchten Kinder lag im Mittel bei 29/3 $\pm 2 / 3$ SSW. Das Geburtsgewicht betrug im Mittel $1200 \pm 288$ g. Die Untersuchungen ergaben im MDI ein mittleres Entwicklungsalter von 23,2 $\pm 4,2$ Monaten. Die motorische Entwicklung (PDI) lag im Mittel bei einem Entwicklungsalter von $21,3 \pm 5$,o Monaten. Einen signifikanten Unterschied gab es zwischen den Geschlechtern.

Schlussfolgerung. Mit dem BSID II lässt sich der Entwicklungsstand mit korrigiertem 2. Lebensjahr objektiv darstellen. Im o. g. Zeitraum gab es in unserem Perinatalzentrum eine deutlich höhere Anzahl weiblicher Frühgeborener $<1500 \mathrm{~g}$. Diese zeigten gegenüber den Jungen ein besseres Outcome im Bereich Kognition und Motorik. Die Chancen auf ein positives Outcome steigen mit Geburtsgewicht und/oder SSW. Die Ergebnisse korrelieren durchaus mit denen größerer Perinatalzentren Level 1. Eine weitere standardisierte Überprüfung des Entwicklungsstandes wäre im Vorschulalter sinnvoll.

\section{DGSPJ-PO-SP-16}

Anwendung des Developmental Coordination Disorder Questionnaire - German (DCDQ - G) in einer Ambulanz für Kinder mit Kommunikationsstörungen

Thiede A.U. ', Fellinger J.'

'Institut für Sinnes- und Sprachneurologie, Konventhospital Barmherzige Brüder Linz, Neurologisch-Linguistische Ambulanz, Linz, Österreich

Fragestellung. Wie schätzen Eltern von Kindern mit Sprachentwicklungsstörungen, Hörstörungen oder Autismus die motorische Entwicklung ihrer Kinder ein? Dafür wurde der DCDQ - G eingesetzt.

Material und Methode. Der DCDQ - G ist ein Elternfragebogen, der als Screening Instrument zur Diagnosestellung von umschriebenen Entwicklungsstörungen motorischer Funktionen (UEMF) entwickelt wurde. Dem Ergebnis des Fragebogens gegenübergestellt wurde die klinische Beurteilung der neuromotorischen Fertigkeiten durch einen Arzt. Eltern von 50 Kindern im Alter von 5.o bis 7.11 Jahren (Durchschnittsalter 5.10 Jahre) füllten den DCDQ - G aus. Davon waren 32 Kinder (Durchschnittsalter 5.8 J.) mit Fragestellung Sprachentwicklung (inkludiert in die Gruppe sind Kinder mit Hörstörungen und Autismus). Ergebnisse. Verglichen wurden die Daten mit den Werten aus der deutschen Studie von Kennedy-Behr et al. von 2013 und der kanadischen Originalstudie von Wilson et al. von 2009. Die Spezifität (SP) und die Sensitivität (SE) in der Gesamtgruppe (SP o,6; SE o,85) und der Testgruppe (SP o,67; SE o,86) mit den Werten der anderen Studien vergleichbar. Die Prävalenz der elterlichen Beurteilung für motorische Entwicklungsauffälligkeiten in der Gesamtgruppe 59\% und in der Testgruppe $58 \%$.

Schlussfolgerung. Der DCDQ - G ist ein ausreichend gutes Screening Instrument zur Einschätzung der motorischen Entwicklung bei Kindern durch die Eltern auch in einer Ambulanz für Kommunikationsstörungen mit Schwerpunkt Sprachentwicklungsstörungen, Hörstörungen und Autismus. Die Ergebnisse sind vergleichbar mit den Daten von Kindern aus Entwicklungsneurologischen Ambulanzen in Deutschland und Kanada.

\section{DGSPJ-PO-SP-17}

Zugang zur stationären Kinderrehabilitation - Probleme aus Perspektive der beteiligten Kinder- und Jugendärzte

Schumann N. ${ }^{1}$, Fach E.-M. ${ }^{1}$, Richter M. ${ }^{1}$

'Institut für Medizinische Soziologie der Martin-Luther-Universität HalleWittenberg, Halle (Saale)

Fragestellung. Kinder- und Jugendärzte sind wichtige Gatekeeper im Zugang zur stationären Kinderrehabilitation. Sinkende Antragszahlen in diesem Versorgungsbereich deuten auf eine Zugangsproblematik 
hin. Ergebnisse aus der Erwachsenenrehabilitation zeigen, dass Ärzte mit verschiedenen Problemen im Zuweisungs- und Antragsverfahren von Rehamaßnahmen konfrontiert sind (Pohontsch et al. 2013; Schubert et al. 2012). Die Frage ist, welche zentralen Probleme sehen Kinderund Jugendärzte im Antragsverfahren von stationären Rehabilitationsmaßnahmen?

Material und Methoden. Niedergelassene Kinder- und Jugendärzte aus Mitteldeutschland ( $\mathrm{N}=82$ ) wurden im Rahmen der rekju-Studie zu Problemen im Antragsverfahren von stationären Kinderrehabilitationsmaßnahmen befragt. Die Ergebnisse wurden deskriptiv ausgewertet. Ergebnisse. Als häufigste Probleme werden die hohe Ablehnungsrate, intransparente Ablehnungsbegründungen trotzt Indikation, fehlende Rückkopplung des Kostenträgers mit dem verantwortlichen Arzt sowie unklare Zugangskriterien und Zuweisungsverfahren (u. a. Trägerzuständigkeit von DRV und GKV) von den Ärzten berichtet.

Diskussion und Schlussfolgerung. Die Ergebnisse zeigen, dass Kinderund Jugendärzte mit verschiedenen Problemen im Antragsverfahren zur stationären Kinderrehabilitation konfrontiert sind, was die Antragszahlen beeinflussen kann. Die Ergebnisse werden in dem aktuell laufenden Projekt KIREZplus mittels Fokusgruppendiskussionen mit Ärzten vertiefend exploriert. Optimierungspotentiale aus Arztperspektive werden herausgearbeitet und sollen dazu beitragen eine trägerorientierte Einbindung der Ärzte in das Antragsverfahren zu fördern und damit auch eine effektive und effiziente Versorgung chronisch kranker Kinder und Jugendlicher sicherzustellen.

\section{DGSPJ-PO-SP-18}

Gelingt eine Verminderung von Kindesvernachlässigung durch Fachkräfte Frühe Hilfen?

\section{Windorfer A.H.R.' \\ 'Stiftung EINE CHANCE FÜR KINDER, Hannover}

Fragestellung. Gelingt durch den Einsatz von Fachkräften Frühe Hilfen (Familienhebammen und Familienkinderkrankenschwestern) eine Verminderung von Kindesvernachlässigung und Kindesmisshandlung?

Material und Methode. Die Stiftung EINE CHANCE FÜR KINDER setzt bei der aufsuchenden Arbeit von Fachkräften Frühe Hilfen eine standardisierte Dokumentation ein; diese erlaubt sowohl eine Qualitätssicherung, wie auch eine gute Evaluation. Die Ergebnisse über den aufsuchenden Einsatz von 140 Fachkräften Frühe Hilfen aus 20 norddeutschen und aus 12 ostdeutschen Kommunen werden vorgestellt.

Ergebnisse. In einigen Kommunen konnten bis zu 7\% der Neugeborenen und Säuglinge über $1 \mathrm{Jahr}$ aufsuchend betreut werden, während es in anderen trotz erheblichen Bedarfs lediglich 1-2\% waren. Trotz der Vorgabe vor allem im Bereich der Sekundärprävention zu arbeiten, war die "Zuweisung" an die Fachkräfte sehr unterschiedlich; nicht mehr als max. 30\% der Betreuungen sollten von einem Jugendamt veranlasst sein; in manchen Kommunen waren dies jedoch $80 \%$. Vor allem sollte die Betreuung so früh wie möglich, bereits am besten während der Schwangerschaft beginnen. Dies war im Durchschnitt in 50\% der Betreuungen möglich. Ein besonderes Augenmerk war auf „Teenie-Mütter“ gerichtet. Entscheidend für den Erfolg waren strukturelle Einbindung der Fachkräfte, fachliches Wissen und begleitende Qualitätssicherung.

Diskussion. Die Einsätze von Fachkräften Frühe Hilfen können Kindesvernachlässigung vermindern helfen, wenn die richtigen Rahmenbedingungen und ein gutes Netzwerk vorliegen. Die Zusammenarbeit mit den vor Ort arbeitenden Kinderärzten ist ein besonders wichtiges Kriterium für das Gelingen.

\section{DGSPJ-PO-SP-19}

Adhärenz zu Cysteamin und klinische Ergebnisse bei 224 US-Patienten mit Cystinose

Greenbaum L.A. ', Lapidus D. ${ }^{2}$, Cadieux B. ${ }^{3}$

${ }^{1}$ Emory University, Atlanta, Vereinigte Staaten von Amerika, ${ }^{2}$ Lapidus Data Inc, Boston, Vereinigte Staaten von Amerika, ${ }^{3}$ Raptor Pharmaceuticals Inc, San Francisco, Vereinigte Staaten von Amerika

Fragestellung. Früher Beginn und Adhärenz (Adh) zu Cysteamin (C) verhindern Progression zur terminaler Niereninsuffizienz und extrarenalen Komplikationen bei Cystinose (Cys). Sofort freigesetztes C (IR-C) erfordert chronische, lebenslange Dosierung, 4-mal täglich alle 6 Stunden. Wir haben Daten von US Krankenversicherungen von Patienten (Pat) mit Cys ausgewertet, um die Adh zu IR-C zu bestimmen und die Therapieergebnisse bei Nieren und anderen Organen zu bewerten.

Methoden. US Krankenversicherung Erstattungen an IR-C Adh bei Cys Pat wurden ausgewertet. Von 2002 bis 2013 identifizierten wir 224 Pat (112 m, o-53 Jahre) mit Cys. Adh wurde von monatlichen Rezepteinlösungen gemessen und eine Rate an Medikamentenbesitz (RMB) berechnet: \% der Monaten mit Einlösungen für IR-C.

Ergebnisse. Von 204 Pat mit >1 Jahr von IR-C Käufen (Æ 5,6 Jahre) hatten 117 Pat (54\%) eine RMB >80\%. Die RMB nahm mit steigendem Alter ab: $86 \%$ (o-5 Jahre, $n=24), 90 \%$ (6-10 Jahre, $n=33$ ), 81\% (11-15; $n=34), 79 \%$ (16-20 Jahre, $n=38$ ), 67\% (Alter $>20$ Jahre, $n=75) .61 / 224$ Pat $(27 \%), 51 \%$ waren jünger als 15 Jahre, hatten keine IR-C Nachkäufe für $>6$ Monate seit dem letzten Kauf, was auf Absetzen der Therapie hindeutet. 45 von 85 Patienten (53\%), die mit Cys im Alter 0-5 diagnostiziert wurden, hatten auch Gedeihstörungen. 40 von 140 (29\%) Pat, welche nach der Einführung von IR-C geboren wurden, entwickelten eine terminale Niereninsuffizienz (Æ 12 Jahre; 5-19 Jahre) und 36 (26\%) Pat. einen Diabetes (13, O-5 Jahre; 9, 6-10 Jahre; 12, 11-15 Jahre; 2, 16-20 Jahre).

Schlussfolgerungen. Adh zu IR-C war niedrig und nahm mit steigendem Alter ab. Weitere Auswertungen zielen auf die Belastung durch die Cystinoseerkrankung und den Zusammenhang von Beginn und Adh einer Behandlung mit IR-C auf die Therapieergebnissen. 